\title{
PreSEIS: A Neural Network-Based Approach to Earthquake Early Warning for Finite Faults
}

\author{
by Maren Böse*, Friedemann Wenzel, and Mustafa Erdik
}

\begin{abstract}
The major challenge in the development of earthquake early warning (EEW) systems is the achievement of a robust performance at largest possible warning time. We have developed a new method for EEW—called PreSEIS (Pre-SEISmic) — that is as quick as methods that are based on single station observations and, at the same time, shows a higher robustness than most other approaches. At regular timesteps after the triggering of the first EEW sensor, PreSEIS estimates the most likely source parameters of an earthquake using the available information on ground motions at different sensors in a seismic network. The approach is based on two-layer feedforward neural networks to estimate the earthquake hypocenter location, its moment magnitude, and the expansion of the evolving seismic rupture. When applied to the Istanbul Earthquake Rapid Response and Early Warning System (IERREWS), PreSEIS estimates the moment magnitudes of 280 simulated finite faults scenarios $(4.5 \leq \mathbf{M} \leq 7.5)$ with errors of less than \pm 0.8 units after $0.5 \mathrm{sec}, \pm 0.5$ units after $7.5 \mathrm{sec}$, and \pm 0.3 units after $15.0 \mathrm{sec}$. In the same time intervals, the mean location errors can be reduced from $10 \mathrm{~km}$ over $6 \mathrm{~km}$ to less than $5 \mathrm{~km}$, respectively. Our analyses show that the uncertainties of the estimated parameters (and thus of the warnings) decrease with time. This reveals a trade-off between the reliability of the warning on the one hand, and the remaining warning time on the other hand. Moreover, the ongoing update of predictions with time allows PreSEIS to handle complex ruptures, in which the largest fault slips do not occur close to the point of rupture initiation. The estimated expansions of the seismic ruptures lead to a clear enhancement of alert maps, which visualize the level and distribution of likely ground shaking in the affected region seconds before seismic waves will arrive.
\end{abstract}

\section{Introduction}

Earthquake early warning (EEW) systems serve as tools for coseismic risk reduction. Basically, they make use of differences in the propagation speed of seismic and electromagnetic waves and issue warnings, if necessary, to potential users before strong shaking at these sites occurs. The maximal warning time of an EEW system is generally defined as the time span between the $P$-wave detection at the first triggered EEW sensor and the arrival of high-amplitude $S$ or surface waves at the user site. As these time periods usually are extremely short, EEW systems must recognize the severity of expected ground motions within a few seconds. Based on this information, suitable actions for the damage reduction can be triggered and executed. Possible measures are, for example, the automatic slowdown of rapid-transit vehicles and high-speed trains to avoid accidents, the automatic shutdown

*Present address: California Institute of Technology, Seismological Laboratory, 1200 E. California Blvd., Mail Code 252-21, Pasadena, California 91125; mboese@gps.caltech.edu. of pipelines and gas lines to minimize fire hazards, the automatic shutdown of manufacturing operations to decrease potential damage to equipment, the automatic saving of vital computer information to avoid losses of data, and actions that support the semiactive control of structures to prevent building collapses (Harben, 1991; Goltz, 2002).

EEW systems shall comply with primary two requirements: they have to be very fast and, at the same time, highly reliable. The two main types of EEW systems, regional and onsite warning systems, meet these claims differently (Kanamori, 2005). Regional warning systems, such as the one installed in Taiwan (Wu and Teng, 2002), use networks of seismic sensors with real-time capability to determine the source parameters of an earthquake. These parameters are then interpreted as a specific warning level. Onsite warning systems, such as the Japanese UrEDAS (Nakamura, 1989), the Californian ElarmS (Allen and Kanamori, 2003), or the Romanian EEW system (Wenzel et al., 1999; Böse et al., 2007), in contrast, are based on seismic observations at sin- 
gle sensors. This characteristic makes them much faster than the regional EEW systems, which can often issue warnings only minutes after the occurrence of an earthquake. In return, onsite EEW systems are less robust than their regional counterpart: high scatter in estimates of source parameters (and thus of warnings) often require averaging over predictions at several onsite warning sensors (Lockman and Allen, 2005; Wu and Kanamori, 2005), which is in contradiction to the initial idea behind onsite warning.

Onsite warning systems generally use only the first few seconds of the $P$-wave signal to estimate the magnitude of an earthquake in progress. This presumes that the earthquake process is deterministic and the final expansion of the rupture thus predetermined (Allen and Kanamori, 2003; Olson and Allen, 2005). Kanamori (2005) suggests the predominant period in the $P$ phase of the seismic displacement record to be an indicator for the earthquake magnitude. This parameter is related to the moment-rate function of the earthquake. Analyzing the data of 52 strong earthquakes $(6.0 \leq \mathbf{M} \leq 8.0)$ recorded by the Japanese Hi-Net seismic network, Rydelek and Horiuchi (2006), however, cannot find an evidence for the hypothesized rupture determinism. Their study suggests that a magnitude cannot be determined until the rupture is completed. Only for smaller events with $\mathbf{M} \leq 6.0$, for which the first few seconds contain almost the entire rupture history, high correlations between the predominant period and the magnitude of an earthquake can be expected and are actually observed (Rydelek and Horiuchi, 2006). Kanamori (2005) proposes to specify the lower bound of the expected earthquake magnitude from the first $3 \mathrm{sec}$ of the $P$-wave signal instead of predicting its final size.

In this article we will present a new method for EEW, called PreSEIS (Pre-SEISmic), that takes advantage of both regional and onsite warning paradigms. At regular timesteps after the triggering of the first sensor, PreSEIS determines the most likely source parameters of an earthquake using the available information on ground shaking at the different sensors in a seismic network. In doing so, PreSEIS includes the entire rupture history of the earthquake, from its beginning to the time at which an estimate is given. Consequently, PreSEIS requires no assumptions on rupture determinism.

We will demonstrate the PreSEIS approach by using the example of the Turkish megacity Istanbul. Within the Istanbul Earthquake Rapid Response and Early Warning System (IERREWS) the Kandilli Observatory of the Bogazici University in Istanbul operates 10 strong-motion sensors with realtime communication links to two datacenters in Istanbul (Erdik, Fahjan, et al., 2003). An EEW system in the Marmara region must be capable of estimating the severity of impending ground shaking within shortest time after the detection: warning times for Istanbul are expected to range between 5 and $25 \mathrm{sec}$ only (Böse, 2006). Istanbul is exposed to a huge seismic risk due to the high concentration of human and industrial settlements in the Marmara region, which are close to seismic fault segments under the Sea of Marmara. These faults form the western continuation of the North Anatolian fault. Some of the segments run only 15 to $20 \mathrm{~km}$ south of Istanbul (Armijo et al., 2002; Le Pichon et al., 2003). From previous earthquakes in the Sea of Marmara and the stress transfer of the 17 August 1999 Izmit earthquake (M 7.4), Parsons (2004) determines a $53 \pm 18 \%$ probability of strong shaking in the Istanbul metropolitan area during the next 30 yr. According to estimates by Erdik, Aydinoglu, et al. (2003), an M 7.5 earthquake in the Marmara region will cause losses of up to 11 billion U.S. dollars, a death total of 40,000 to 50,000 , and the destruction of 430,000 to 600,000 households.

\section{Method}

Both the earthquake rupture and the propagation of seismic waves are time-dependent processes that provide timedependent information on the current earthquake. This information is contained in ground shaking at the sensors of a seismic network from which we can derive certain parameters. Throughout this article, we will call these parameters seismic attributes. The consideration of time dependency is essential for the design of PreSEIS: at regular timesteps $n=$ $1, \ldots, N$ after the detection of an earthquake, PreSEIS determines the most likely source parameters of the event using the available information on ground shaking at different sensors in a seismic network. Only $0.5 \mathrm{sec}$ after the $P$-wave detection at the first sensor, PreSEIS starts to issue estimates of the hypocenter location (characterized by the geographical latitude $\Phi$, longitude $\Theta$, and source depth $Z$ ), the moment magnitude $\mathbf{M}$, and the expansion of the evolving earthquake rupture. We presume a rectangular rupture plane, which is characterized by the geographical coordinates of the spatial start and end points of the rupture given by ( $\Phi_{\text {start }}$, $\left.\Theta_{\text {start }}, \Phi_{\text {end }}, \Theta_{\text {end }}\right)$. With ongoing time, longer time series of ground motion at more and more sensors become available and allow for a regular update of the estimated source parameters $\left(\hat{\Phi}^{n}, \hat{\Theta}^{n}, \hat{Z}^{n}\right), \hat{M}^{n}$, and $\left(\hat{\Phi}_{\text {start }}^{n}, \hat{\Theta}_{\text {start }}^{n}, \hat{\Phi}_{\text {end }}^{n}, \hat{\Theta}_{\text {end }}^{n}\right)$ (e.g., every half second).

In the following, we will assume that we have a seismic network of $I$ properly working ground-motion sensors. From the sets of observed ground motions at each sensor $i$ $(i=1, \ldots, I)$ we derive two types of seismic attributes: (1) the time difference $\Delta \tau_{i}=\tau_{i}-\tau_{i_{0}}$ between the $P$-wave arrival at the $i$ th sensor, $\tau_{i}$, and at the first triggered sensor $i_{0}$, $\tau_{i_{0}}$, and (2) the cumulative absolute velocity (CAV, Kramer, 1996) $\mathrm{CAV}_{i}$ obtained from the time integration of ground acceleration at sensor $i$. Note that the seismic attributes are time-dependent parameters that require the introduction of a new index $n(n=1, \ldots, N)$ in our notations as a marker for the respective timestep. We assign $n=1$ to the first timestep after triggering of the first EEW sensor in the network and stop our analyses after $n=N$ steps. Details on the two attribute sets $\Delta \tau_{i}^{n}$ and $\mathrm{CAV}_{i}^{n}$ will be given in the following paragraphs.

Within the first few seconds after the rupture initiation of an earthquake, the $P$ wave will generally have arrived at a 
minor subset of sensors, implying that the inversion problem for the hypocenter location will likely be ill posed. Including the additional information on nontriggered sensors, at which the $P$ wave has not yet arrived, however, allows confining the space of possible solutions, because we can rule out certain positions (Rydelek and Pujol, 2004; Horiuchi et al., 2005). At a given time $t^{n}$ we can, of course, specify $\tau_{i}$ only for those sensors at which the $P$ wave has already arrived (i.e., $\left.\tau_{i} \leq t^{n}\right)$. If, on the other hand, a sensor is not triggered at time $t^{n}$, it follows that $\tau_{i}>t^{n}$ (assuming that the system has not missed the $P$-wave onset). For the specification of the time difference between the $P$-wave arrival at the $i$ th sensor in the network and the first triggered sensor $i_{0}$ at time $t^{n}$, we introduce the following definition:

$$
\Delta \tau_{i}^{n} \equiv\left\{\begin{array}{ll}
t^{n}-\tau_{i_{0}}, & \tau_{i}>t^{n} \\
\tau_{i}-\tau_{i_{0}}, & \tau_{i} \leq t^{n},
\end{array} \quad n=1, \ldots, N\right.
$$

where $\tau_{i_{0}} \leq \tau_{i}$ for $i=1, \ldots, I$. The first relation in equation (1) gives the minimum time difference for a sensor at which the $P$ wave has not arrived at time $t^{n}$; the second relation gives the measured time difference for a sensor that already has detected the $P$ wave. Aside from the information that the $P$ wave has not reached certain sensors, PreSEIS also includes the a priori information on likely source locations. Both help to confine the space of possible source locations (see explanation later in this article).

The second parameter set holds the values of the cumulative absolute velocity $\mathrm{CAV}_{i}^{n}$ that are derived from the mean horizontal component of the band pass-filtered acceleration time series (here $0.05-12.0 \mathrm{~Hz}$ ) at each sensor $i=1, \ldots, I$ at timestep $n(n=1, \ldots, N)$ after $\tau_{i_{0}}$. To account for the large bandwidth of CAV levels for different earthquakes, we take their logarithmic values and define

$$
\begin{aligned}
& \log \left(\mathrm{CAV}_{i}^{n}+1\right) \equiv \begin{cases}0, & \tau_{i}>t^{n} \\
\log \left(\sum_{t=\tau_{i}}^{t^{n}}\left|a_{i}(t)\right| d t+1\right), & \tau_{i} \leq t^{n},\end{cases} \\
& n=1, \ldots, N,
\end{aligned}
$$

where $\left|a_{i}(t)\right|$ is the absolute amplitude of ground acceleration in $\left(\mathrm{cm} / \mathrm{sec}^{2}\right)$ observed at the $i$ th sensor at time $t ; d t$ is the sampling interval of the ground-motion time series. The CAV at a sensor that is not triggered at time $t^{n}$ (i.e., $\tau_{i}>t^{n}$ ) is set to zero. Because the logarithm is not defined at zero, we add a constant factor of $1(\mathrm{~cm} / \mathrm{sec})$.

For the inversion of seismic source parameters $(\Phi, \Theta$, $Z), \mathbf{M}$, and $\left(\Phi_{\text {start }}, \Theta_{\text {start }}, \Phi_{\text {end }}, \Theta_{\text {end }}\right)$ from the sets of time-dependent seismic attributes $\Delta \tau_{i}^{n}$ and $\log \left(\mathrm{CAV}_{i}^{n}+1\right)$, PreSEIS makes use of artificial neural networks (ANNs). ANNs provide statistical models for complex (nonlinear) processes and relations (Bishop, 1995). They are characterized by a massively parallel structure with high numbers of simple processing units called neurons that are connected to each other.
The importance of the link between two neurons is controlled by a weight parameter. The weight parameters of an ANN are iteratively adapted to the inversion problem by learning from a set of example patterns with known input and known output values (supervised learning). Once this training procedure is accomplished, ANNs are capable of processing unknown data that have the statistical properties of the training examples. Advantageous features of ANNs are their independence from formulations of explicit algorithms and their high tolerance of noisy input data (Bishop, 1995).

In PreSEIS we apply ANNs for the recognition of patterns of seismic attributes and their statistical association with specific source parameters. We use two-layer feed-forward (TLFF) neural networks, in which the neurons are arranged in three layers: one input, one hidden, and one output layer (Fig. 1). The outputs of TLFF networks are explicit functions of the input values and the weight parameters $w_{j i}^{(1)}$ and $w_{k j}^{(2)}$ in the hidden and output layers $(i=0, \ldots, I$; $j=0, \ldots, J ; k=1, \ldots, K)$. For each timestep $n$ we design three TLFF networks: the first network is used for the estimation of the hypocenter location $\left(\hat{\Phi}^{n}, \hat{\Theta}^{n}, \hat{Z}^{n}\right)$ using the information on time delays of $P$-wave arrivals $\Delta \tau_{i}^{n}$ at the different sensors $i=1, \ldots, I$ using equation (1):

$$
\begin{aligned}
\hat{\Phi}^{n} & =\sum_{j=0}^{J} w_{1 j}^{\operatorname{loc}(2), n} g\left(\sum_{i=0}^{I} w_{j i}^{\operatorname{loc}(1), n} \Delta \tau_{i}^{n}\right), \\
\hat{\Theta}^{n} & =\sum_{j=0}^{J} w_{2 j}^{\operatorname{loc}(2), n} g\left(\sum_{i=0}^{I} w_{j i}^{\operatorname{loc}(1), n} \Delta \tau_{i}^{n}\right),
\end{aligned}
$$

and

$$
\hat{Z}^{n}=\sum_{j=0}^{J} w_{3 j}^{\operatorname{loc}(2), n} g\left(\sum_{i=0}^{I} w_{j i}^{\operatorname{loc}(1), n} \Delta \tau_{i}^{n}\right),
$$

where $g(\cdot)$ is the logistic sigmoid function defined by

$$
g(\arg ) \equiv \frac{1}{1+\exp (-\arg )} .
$$

This function maps the argument (arg) into the interval $(0,1)$ and introduces a nonlinear element into the transfer functions (see equations $3 \mathrm{a}, 3 \mathrm{~b}$, and $3 \mathrm{c}$ ).

The second network uses the outputs $\left(\hat{\Phi}^{n}, \hat{\Theta}^{n}, \hat{Z}^{n}\right)$ of the first network along with the amplitude information $\log \left(\mathrm{CAV}_{i}^{n}+1\right)$, computed using equation (2), to predict the moment magnitude $\hat{M}^{n}$ of the earthquake at timestep $n$ :

$$
\begin{aligned}
\hat{M}^{n}= & \sum_{j=0}^{J} w_{1 j}^{\operatorname{mag}(2), n} g\left(\sum_{i=0}^{I} w_{j i}^{\operatorname{mag}(1), n} \log \left(\mathrm{CAV}_{i}^{n}+1\right)\right. \\
& \left.+w_{j(I+1)}^{\operatorname{mag}(1), n} \hat{\Phi}^{n}+w_{j(I+2)}^{\operatorname{mag}(1), n} \hat{\Theta}^{n}+w_{j(I+3)}^{\operatorname{mag}(1), n} \hat{Z}^{n}\right) .
\end{aligned}
$$

Finally, the third network estimates the geographical coordi- 
(a)

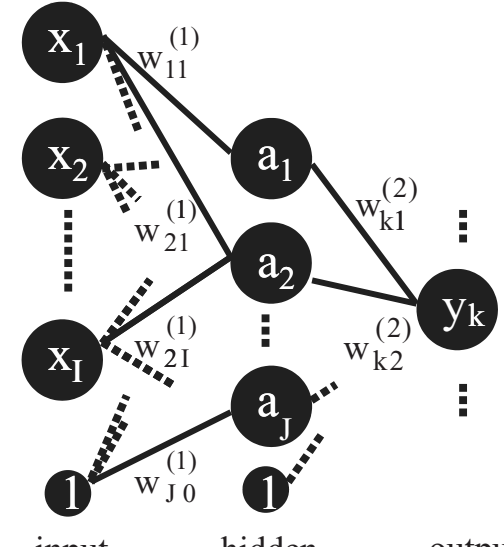

(b)

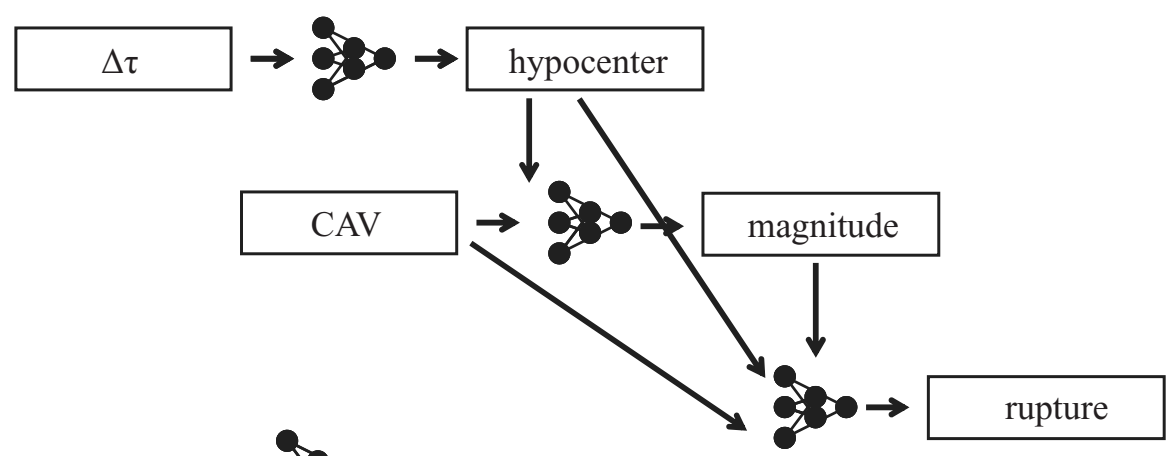

input hidden output

Figure 1. (a) Scheme of a two-layer feed-forward (TLFF) neural network composed of one input, one hidden, and one output layer. All neurons of subsequent layers are connected to each other by a network of weighted links, which are iteratively adapted to the mapping $\mathrm{X} \rightarrow \mathrm{Y}$ from a set of example patterns. (b) PreSEIS applies three TLFF networks for the estimation of seismic source parameters. The first network uses the information on the time differences $\Delta \tau$ between the $P$-wave arrivals at the different sensors to estimate the location of the earthquake hypocenter. The second uses the CAV and the estimated hypocenter location to estimate the moment magnitude. The third network, finally, uses the outputs of the other two networks along with the CAV values in order to predict the expansion of the evolving seismic rupture.

nates of the rupture start and end points $\left(\hat{\Phi}_{\text {start }}^{n}, \hat{\Theta}_{\text {start }}^{n}\right.$, $\left.\hat{\Phi}_{\text {end }}^{n}, \hat{\Theta}_{\text {end }}^{n}\right)$ using the outputs of the previous two networks combined with the cumulative absolute velocity $\log \left(\mathrm{CAV}_{i}^{n}+\right.$ 1) at the different sensors $i=1, \ldots, I$ :

$$
\begin{aligned}
\hat{\Phi}_{\mathrm{start}}^{n}= & \sum_{j=0}^{J} w_{1 j}^{\operatorname{rup}(2), n} g\left(\sum_{i=0}^{I} w_{j i}^{\operatorname{rup}(1), n} \log \left(\mathrm{CAV}_{i}^{n}+1\right)\right. \\
& +w_{j(I+1)}^{\operatorname{rup}(1), n} \hat{\Phi}^{n}+w_{j(I+2)}^{\operatorname{rup}(1), n} \hat{\Theta}^{n}+w_{j(I+3)}^{\operatorname{rup}(1), n} \hat{Z}^{n} \\
& \left.+w_{j(I+4)}^{\operatorname{rup}(1), n} \hat{M}^{n}\right), \\
\hat{\Theta}_{\text {start }}^{n}= & \sum_{j=0}^{J} w_{2 j}^{\operatorname{rup}(2), n} g\left(\sum_{i=0}^{I} w_{j i}^{\operatorname{rup}(1), n} \log \left(\mathrm{CAV}_{i}^{n}+1\right)\right. \\
& +w_{j(I+1)}^{\operatorname{rup}(1), n} \hat{\Phi}^{n}+w_{j(I+2)}^{\operatorname{rup}(1), n} \hat{\Theta}^{n}+w_{j(I+3)}^{\operatorname{rup}(1), n} \hat{Z}^{n} \\
& \left.+w_{j(I+4)}^{\operatorname{rup}(1), n} \hat{M}^{n}\right), \\
& \left.+w_{j(I+4)}^{\operatorname{rup}(1), n} \hat{M}^{n}\right), \\
\hat{\Phi}_{\mathrm{end}}^{n}= & \sum_{j=0}^{J} w_{3 j}^{\operatorname{rup}(2), n} g\left(\sum_{i=0}^{I} w_{j i}^{\operatorname{rup}(1), n} \log \left(\mathrm{CAV}_{i}^{n}+1\right)\right. \\
& +w_{j(I+1)}^{\operatorname{rup}(1), n} \hat{\Phi}^{n}+w_{j(I+2)}^{\operatorname{rup}(1), n} \hat{\Theta}^{n}+w_{j(I+3)}^{\operatorname{rup}(1), n} \hat{Z}^{n} \\
&
\end{aligned}
$$

and

$$
\begin{aligned}
\hat{\Theta}_{\text {end }}^{n}= & \sum_{j=0}^{J} w_{4 j}^{\operatorname{rup}(2), n} g\left(\sum_{i=0}^{I} w_{j i}^{\operatorname{rup}(1), n} \log \left(\mathrm{CAV}_{i}^{n}+1\right)\right. \\
& +w_{j(I+1)}^{\operatorname{rup}(1), n} \hat{\Phi}^{n}+w_{j(I+2)}^{\operatorname{rup}(1), n} \hat{\Theta}^{n}+w_{j(I+3)}^{\operatorname{rup}(1), n} \hat{Z}^{n} \\
& \left.+w_{j(I+4)}^{\operatorname{rup}(1), n} \hat{M}^{n}\right) .
\end{aligned}
$$

According to equations (3a) to (3c), (5), and (6a) to (6d), each source parameter and each timestep $n$ has its own TLFF network. Because the seismic attributes and the earthquake source parameters are not independent of each other, we link the inputs and outputs of the three networks as shown in Figure 1b. A smoothing average procedure over the outputs of the TLFF networks at different timesteps $n$ eliminates possible outliers in the estimates.

According to the transfer functions (3a) to (3c), (5), and (6a) to (6d), the outputs of each TLFF network are computed from the nested linear combinations of seismic attributes (where $\Delta \tau_{0}^{n} \equiv 0$ and $\mathrm{CAV}_{0}^{n} \equiv 0$ ) and weight parameters

$$
\begin{gathered}
\mathbf{w}^{\operatorname{loc}, n}=\left\{w_{j i}^{\operatorname{loc}(1), n}, w_{1 j}^{\operatorname{loc}(2), n}, w_{2 j}^{\operatorname{loc}(2), n}, w_{3 j}^{\operatorname{loc}(2), n}\right. \\
\mid i=0, \ldots, I ; j=0, \ldots, J\},
\end{gathered}
$$


$\mathbf{w}^{\mathrm{mag}, n}=\left\{w_{j i}^{\operatorname{mag}(1), n}, w_{1 j}^{\operatorname{mag}(2), n} \mid i=0, \ldots, I+3 ; j=0, \ldots, J\right\}$,

and

$$
\begin{gathered}
\mathbf{w}^{\text {rup }, n}=\left\{w_{j i}^{\operatorname{rup}(1), n}, w_{1 j}^{\operatorname{rup}(2), n}, w_{2 j}^{\operatorname{rup}(2), n}, w_{3 j}^{\operatorname{rup}(2), n}, w_{4 j}^{\operatorname{rup}(2), n}\right. \\
\mid i=0, \ldots, I+4 ; j=0, \ldots, J\} .
\end{gathered}
$$

The total number of weights $W$ in a TLFF network depends on the number of neurons in the input, the hidden, and the output layer- $I, J$, and $K$-respectively, and is given by

$$
W=(I+1) \cdot J+(J+1) \cdot K \text {. }
$$

In our three networks we have $K=3, K=1$, and $K=4$ output values using equations (3a) to (3c), (5), and (6a) to (6d), respectively. The model complexity of TLFF networks is mainly controlled by the number $J$ of neurons in the hidden layer. The optimum number of $J$ is usually determined through systematic tests of different network architectures (Swingler, 1996). For the design discussed in this article, we achieved optimum performance for $J=6$ (Böse, 2006).

To determine the weight parameters defined by equations (7a) to (7c), we use a set of $S$ earthquakes with known locations, magnitudes, and rupture histories. Using this training set, we minimize the following sums of squared errors (SSE) between the known output and that obtained for a given set of parameters:

$$
\begin{aligned}
& \operatorname{SSE}^{\mathrm{loc}, n}\left(\mathbf{w}^{\mathrm{loc}, n}\right) \\
& \equiv \sum_{s=1}^{S}\left[\left(\Phi_{s}-\hat{\Phi}_{s}^{n}\right)^{2}+\left(\Theta_{s}-\hat{\Theta}_{s}^{n}\right)^{2}+\left(Z_{s}-\hat{Z}_{s}^{n}\right)^{2}\right] \\
& \operatorname{SSE}^{\mathrm{mag}, n}\left(\mathbf{w}^{\mathrm{mag}, n}\right) \equiv \sum_{s=1}^{S}\left[\mathbf{M}_{s}-\hat{M}_{s}^{n}\right]^{2}
\end{aligned}
$$

and

$$
\begin{aligned}
& \operatorname{SSE}^{\text {rup }, n}\left(\mathbf{w}^{\text {rup }, n}\right) \\
& \equiv \sum_{s=1}^{S}\left[\left(\Phi_{\text {start }, s}-\hat{\Phi}_{\text {start }, s}^{n}\right)^{2}+\left(\Theta_{\text {start }, s}-\hat{\Theta}_{\text {start }, s}^{n}\right)^{2}\right. \\
& \left.+\left(\Phi_{\text {end }, s}-\hat{\Phi}_{\text {end }, s}^{n}\right)^{2}+\left(\Theta_{\text {end }, s}-\hat{\Theta}_{\text {end }, s}^{n}\right)^{2}\right]
\end{aligned}
$$

The minimization of equations (9a) to (9c) is carried out with respect to the weight parameters $\mathbf{w}^{\text {loc, } n}, \mathbf{w}^{\text {mag, } n}$, and $\mathbf{w}^{\text {rup, } n}$ and is performed using the Levenberg optimization method (LOM) (Levenberg, 1944). The LOM is a heuristic method that combines the common first order gradient descent optimization and the second order Newton's method for iterative weight updates. The required gradients of the error functions with respect to the weight parameters are calcu- lated using a backpropagation algorithm (Rumelhart et al., 1986).

The training starts with a random weight initialization and is terminated once the error for an independent validation subset increases. This early stopping rule has the purpose to avoid undesired overfitting to the training data at the expense of the generalization capability of the ANNs (Bishop, 1995). The trade-off is comparable to the problem of curve fitting: the higher the number of degrees of freedom in the approximating function (i.e., the higher the order of the polynomial), the greater its flexibility and the better the fit to the input data. However, because the data may be affected by noise, a good polynomial fit to the data may result in a poor approximation of the underlying function, which is likely to be represented by a smoother polynomial.

So far, we have described a method that enables us to estimate the source parameters of an earthquake based on the (incomplete) time-dependent information on ground shaking at several sensors in a seismic network. This information is insufficient for a user in the endangered region, close to the earthquake epicenter. The level of ground shaking (in terms of ground-motion parameter $I M$ ), which this user might likely experience, can be estimated from the combination of the source parameters with empirical attenuation laws (e.g., for seismic intensity). In a simple form, we can express $I M$ at site $(\lambda, \theta)$ by a function $f(\cdot)$ with

$$
\operatorname{IM}(\lambda, \theta)=f(\mathbf{M}, d, \text { site })
$$

where $\mathbf{M}$ is the earthquake (moment) magnitude and $d$ a measure of the distance between the earthquake source and site $(\lambda, \theta)$. The third argument in (10a) accounts for the respective site condition at $(\lambda, \theta)$, which usually has a large impact on IM. Empirical attenuation laws are usually determined from regression analyses of regional earthquake data. For EEW we replace $\mathbf{M}$ and $d$ in equation (10a) by $\hat{M}^{n}$ and $\hat{d}_{\text {rup }}^{n}$ (i.e., by the estimated moment magnitude and the estimated Joyner-Boore distance). The later is defined as the closest distance from a site to the surface projection of the fault rupture. We thus obtain the estimated level of ground-shaking $\widehat{I M}^{n}$ at site $(\lambda, \theta)$ at timestep $n$ from the relation

$$
\widehat{I M}^{n}(\lambda, \theta)=f\left(\hat{M}^{n}, \hat{d}_{\text {rup }}^{n}, \text { site }\right),
$$

where $\hat{d}_{\text {rup }}^{n}$ is determined from the distance between sites $(\lambda, \theta)$ and $\left(\hat{\phi}^{n}, \hat{\varphi}^{n}\right)$ given by

$$
\begin{aligned}
\hat{\phi}^{n}= & \hat{\Phi}_{\text {start }}^{n} \\
+ & \frac{\left(\lambda-\hat{\Phi}_{\text {start }}^{n}\right)\left(\hat{\Phi}_{\text {end }}^{n}-\hat{\Phi}_{\text {start }}^{n}\right)+\left(\theta-\hat{\Theta}_{\text {start }}^{n}\right)\left(\hat{\Theta}_{\text {end }}^{n}-\hat{\Theta}_{\text {start }}^{n}\right)}{\left(\hat{\Phi}_{\text {start }}^{n}-\hat{\Phi}_{\text {end }}^{n}\right)^{2}+\left(\hat{\Theta}_{\text {start }}^{n}-\hat{\Theta}_{\text {end }}^{n}\right)^{2}} \\
& \left(\hat{\Phi}_{\text {end }}^{n}-\hat{\Phi}_{\text {start }}^{n}\right),
\end{aligned}
$$


and

$$
\begin{aligned}
\hat{\varphi}^{n}= & \hat{\Theta}_{\text {start }}^{n} \\
+ & \frac{\left(\lambda-\hat{\Phi}_{\text {start }}^{n}\right)\left(\hat{\Phi}_{\text {end }}^{n}-\hat{\Phi}_{\text {start }}^{n}\right)+\left(\theta-\hat{\Theta}_{\text {start }}^{n}\right)\left(\hat{\Theta}_{\text {end }}^{n}-\hat{\Theta}_{\text {start }}^{n}\right)}{\left(\hat{\Phi}_{\text {start }}^{n}-\hat{\Phi}_{\text {end }}^{n}\right)^{2}+\left(\hat{\Theta}_{\text {start }}^{n}-\hat{\Theta}_{\text {end }}^{n}\right)^{2}} \\
& \left(\hat{\Theta}_{\text {end }}^{n}-\hat{\Theta}_{\text {start }}^{n}\right) .
\end{aligned}
$$

Institutions or companies that operate or rely on networks of infrastructure, such as electricity, water supply, or transportation, are not only interested in receiving information on ground shaking at a single site $(\lambda, \theta)$. They more likely need information on the distribution of ground shaking on an urban or on a regional scale. This information can provide a rapid overview of the possible impact of an earthquake on the entire network seconds before the seismic waves will arrive. Maps visualizing the level of likely ground shaking are usually called alert maps (Allen, 2007). They are similar to shake maps (e.g., Wald et al., 1999), but are obtained from the interpolation of $\widehat{I M}^{n}\left(\lambda_{i}, \theta_{i}\right)$ instead of $\operatorname{IM}\left(\lambda_{i}, \theta_{i}\right)$ over different sites $\left(\lambda_{i}, \theta_{i}\right)$. Ideally, the difference between alert and shake maps is small.

The level at which ground shaking becomes critical and warning is needed is user specific. The introduction of userspecific thresholds for $I M$ allows the assignment of a certain warning level to each level of ground shaking. In this article we will use solely one warning level $I M_{\text {thres }}$, which means that we distinguish between the two cases

$$
\widehat{I M}^{n} \geq I M_{\text {thres }},
$$

that is, the expected level of ground shaking exceeds the threshold and the user requires a warning, and

$$
\widehat{I M}^{n}<I M_{\text {thres }},
$$

that is, the earthquake is likely harmless and an alert unnecessary. We define the following:

1. A correct alert means that an earthquake that satisfies equation (11a) is recognized.

2. A missed alert means that an earthquake that satisfies equation (11a) is not recognized.

3. A correct no alert means recognition of an earthquake that satisfies equation (11b).

4. A false alert means an erroneous warning in the case of an earthquake that satisfies equation (11b).

An ideal EEW system will encounter cases (1) and (3) only.

\section{Database}

We will demonstrate the PreSEIS approach in this article by using the example of the Istanbul EEW system as part of the IERREWS (Erdik, Fahjan, et al., 2003). As in many other areas of the world that are affected by a high seismic threat, the development and verification of a method for EEW in the Marmara region is hampered by the lack of moderate and strong-motion data. Note that the IERREWS was installed in 2002/2003. Since then, no significant earthquake has occurred in the Marmara region, which could have been recorded by the stations. We will therefore make use of simulated ground-motion time series for a large suite of possible earthquake scenarios in the Marmara region by application of the stochastic simulation method for finite faults (SSMFF) (Beresnev and Atkinson, 1997). Short-period motions are frequently treated as stochastic phenomena (e.g., Boore, 1983). The SSMFF represents a simple and effective tool for their simulation without requiring detailed specifications of source and subsurface models. The method needs the description of the Fourier amplitude spectrum (FAS) of seismic ground motion as well as simple models that describe its modification due to wave propagation and local site effects. In the following, we will briefly describe two methods for the stochastic modeling of ground motions for point and finite sources.

At large source-to-site distances (or small earthquakes), we can approximate the seismic rupture as a point source. The stochastic point source approach (SPSA) for seismic $S$ waves combines the estimated FAS of ground motion $\left|A_{S}(\mathbf{x}, \omega)\right|$ at a site $\mathbf{x}$ and angular frequency $\omega$, given by

$$
\begin{aligned}
\left|A_{S}(\mathbf{x}, \omega)\right|= & \omega^{2} C_{S} M_{0}\left[1+\left(\frac{\omega}{\omega_{c}}\right)^{2}\right]^{-1} \\
& \times \exp \left(-\frac{\omega d(\mathbf{x})}{2 Q_{S} \beta}\right) G(\mathbf{x}) F(\mathbf{x}, \omega) H(\mathbf{x}, \omega),
\end{aligned}
$$

with a random phase that is obtained from a time series of windowed Gaussian noise of finite duration (Boore, 1983). The first factor in equation (12a) is the well-known $\omega^{2}$ source spectrum after Brune $(1970,1971)$ with corner frequency $\omega_{c}$ and seismic moment $M_{0}$. The spectral constant $C_{S}$, equal to

$$
C_{S} \equiv \frac{f_{S}}{\sqrt{2}} \frac{R_{S}^{\theta \Phi}}{4 \pi \rho \beta^{3}},
$$

depends on the free-surface amplification $f_{S}$ (here $\left.f_{S}=2.0\right)$, the average radiation pattern $R_{S}^{\theta \Phi}(0.55$; Boore and Boatwright, 1984), density $\rho$, and $S$-wave velocity $\beta$. The exponential factor in equation (12a) accounts for the inelastic attenuation of the seismic waves at source-to-site distance $d$ and is quantified by the quality factor $Q_{s}$. The factors $G(\mathbf{x}), F(\mathbf{x}, \omega)$, and $H(\mathbf{x}, \omega)$ account for the geometrical spreading, the site amplification, and the high-frequency diminution of the seismic waves at the site of observation, respectively. A detailed description of the SPSA is given by Boore (2003).

Based on the observation that $S$ and $P$ waves basically have similar spectral features (Boore, 1986), we can extend the SPSA from seismic $S$ to $P$ waves. The latter are generally 
more important for EEW because of their higher velocities. We describe the FAS for seismic $P$ waves $\left|A_{P}(\mathbf{x}, \omega)\right|$ at a site $\mathbf{x}$ and angular frequency $\omega$ with an expression similar to equation (12a), but replace the spectral constant $C_{S}$ in equation (12b) by

$$
C_{P} \equiv \sin (\varepsilon) \frac{f_{P}}{\sqrt{2}} \frac{R_{P}^{\theta \Phi}}{4 \pi \rho \alpha^{3}},
$$

where $\varepsilon$ is the angle of incidence of the seismic $P$ wave and $f_{P}$ is the free surface amplification. We set $f_{P}=2.0$, which is a reasonable value for plane $P$ waves (Jiang et al., 1998). The average radiation pattern for $P$ waves is $R_{P}^{\theta \Phi}=0.33$ (Boore and Boatwright, 1984). The $P$-wave velocity $\alpha$ and the quality factor $Q_{P}$ are assumed to equal $\alpha=\sqrt{3} \beta$ and $Q_{P}=9 / 4 Q_{S}$, respectively. Following Boore (1986), we assume that we have no shift in the corner frequency between $P$ and $S$ waves.

While the SPSA allows the simulation of seismic ground motions at large source-to-site distances, the SSMFF, which we use here, enables us to consider the expansion of the seismic source. This extension is strongly needed for the simulation of seismic ground motions at short source-to-site distances, such as those in the Marmara region. The SSMFF considers the seismic rupture as a system composed of numerous point sources, each spectrally characterized by equation (12a). We obtain the output time series of ground motions at a given site $\mathbf{x}$ through the summation of contributions of all subfault elements with respective time delays as a consequence of the rupture propagation. Details on the SSMFF procedure are given by Beresnev and Atkinson (1997).

With the SSMFF (extended to $P$ waves) we generate a set of synthetic ground-motion records at all 10 EEW stations of the IERREW system and at one additional site close to Istanbul, named UserX (Fig. 2). For the required description of the source, propagation and site models in the Marmara region we take parameters published by Atkinson and Boore (1995), Horasan et al. (1998), and Pulido et al. (2004). The synthetic database contains 280 scenarios with moment magnitudes $4.5 \leq \mathbf{M} \leq 7.5$. Note that the source spectra of ground motions for finite faults are sensitive to seismic $P$ and $S$-wave speed $\alpha$ and $\beta$, as well as to the maximum slip and rupture velocities $v_{\max }$ and $v_{r}$ (Beresnev, 2001). We use simple homogeneous velocity models with $\alpha=5.7 \mathrm{~km} / \mathrm{sec}$, $\beta=3.3 \mathrm{~km} / \mathrm{sec}$, and $v_{r}=0.8 \beta$. We keep source parameters, such as $v_{\max }$, variable within physically reasonable limits to obtain a high degree of aleatory variability in our simulations (Böse, 2006). Because the site effects at the EEW sensors of the IERREWS have not been assessed yet, we make use of the available National Earthquake Hazards Reduction Program (NEHRP) soil classification maps for the Istanbul metropolitan area (Erdik, Aydinoglu, et al., 2003). For $F(\mathbf{x}, w)$ in equation $(12 \mathrm{a})$ we insert mean spectral amplification factors from Boore and Joyner (1997) that are representative for the respective soil class. The database has bi- and unidirectional ruptures with random slip distributions along five fault segments in the Sea of Marmara. Thirty additional earthquakes with $4.5 \leq \mathbf{M} \leq 5.0$ are randomly distributed in the Marmara region. The epicenters of all 280 earthquakes are shown in Figure 2.

Using an empirical relationship between the FAS of ground shaking and the seismic intensity MMI (Sokolov, 2002), we determine the $M M I$ of each simulation. We then establish from regression an empirical attenuation law for seismic intensity in the Marmara region that is consistent with the simulated data. Replacing the ground-motion parameter $I M$ and the estimate $\widehat{I M}^{n}$ in the general equations (10a) and (10b) by $M M I$ and $\widehat{M M I} I^{n}$, respectively, we can estimate the seismic intensity at site $(\lambda, \theta)$ for a given earthquake of moment magnitude $\mathbf{M}$ from

$$
\begin{aligned}
\operatorname{MMI}(\lambda, \theta)= & \exp (1.2655+0.2089 \mathbf{M} \\
& -0.2451 \log \left(d_{\text {rup }}+2.1502 \mathbf{M}\right) \\
& \left.-0.0011 d_{\text {rup }}\right),
\end{aligned}
$$

or for EEW from

$$
\begin{aligned}
\widehat{M M} I^{n}(\lambda, \theta)= & \exp \left(1.2655+0.2089 \hat{M}^{n}\right. \\
& -0.2451 \log \left(\hat{d}_{\text {rup }}^{n}+2.1502 \hat{M}^{n}\right) \\
& \left.-0.0011 \hat{d}_{\text {rup }}^{n}\right)
\end{aligned}
$$

where $d_{\text {rup }}$ and $\hat{d}_{\text {rup }}^{n}$ are the true and the estimated JoynerBoore distances (in kilometers), respectively. Equations (14a) and (14b) refer to rock sites.

In order to build a robust ANN model for a given inversion problem, each free parameter in the network requires about 10 training examples (Bishop, 1995). Using equation (8) with $J=6, I=10, I=13$, and $I=14$, respectively, and $K=3, K=1$, and $K=4$ (depending on the seismic source parameter that shall be estimated), we obtain between 87 and 118 weight parameters. This means that we will need at least 870 to 1180 training examples. Consequently, our simulated database of 280 scenarios is insufficient. We enlarge this set artificially by manipulating the available data. This is a common trick in neural computations: by taking existing training examples and perturbing them, adding noise or transforming the data in some way, it is possible to allow for novel situations that are not covered by the training data (Swingler, 1996). The artificial extension of the available data does therewith not only provide a larger training set, but it will often also enhance the generalization properties of the ANN models. We manipulate our database through two procedures: first, through the introduction of events with erroneously picked $P$-wave onsets with random delays of up to $1 \mathrm{sec}$. Second, we add Gaussian noise of different amplitudes between $\pm 2 \mathrm{~cm} / \mathrm{sec}^{2}$ and $\pm 10 \mathrm{~cm} / \mathrm{sec}^{2}$ to the simulated acceleration time series before calculating the CAV values in equation (2). From the perturbation of the seis- 


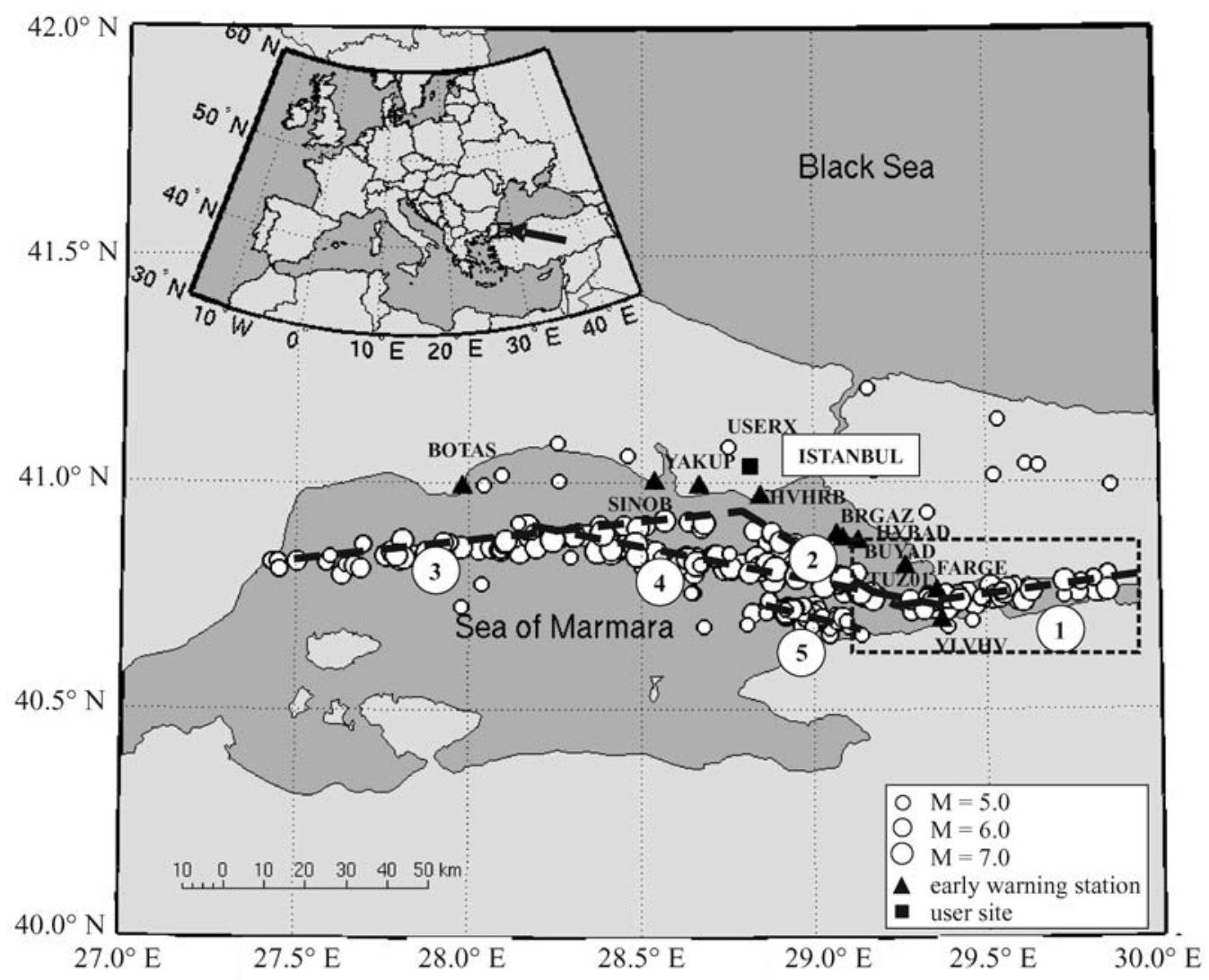

Figure 2. Epicenters of 280 stochastically simulated earthquakes for finite sources $(4.5 \leq \mathbf{M} \leq 7.5)$ in the Sea of Marmara: 50 events occur along the Izmit fault (1), 50 along the Cinarcik fault (2), and 50 along the Western fault (3). Fifty earthquakes are simulated along fault (4), which represents joint ruptures of segments (1) to (3), and 50 along fault (5), which runs through the southeastern part of the Sea of Marmara. The 30 remaining events with $4.5 \leq \mathbf{M} \leq 5.0$ are randomly distributed in the Marmara region. The EEW sensors of the IERREWS (Erdik, Fahjan, et al., 2003) are marked by triangles; the industrial facility UserX-as a possible recipient of warnings-is indicated by a square.

mic attributes, we obtain a new database that includes (1) the original set, (2) five sets with erroneous $P$-wave picks, and (3) five sets with different levels of Gaussian noise. The new database with $N=(1+5+5) 280=3080$ patterns is 11 times larger than the initial set.

\section{Results}

The following results are based on the station configuration of EEW sensors in the IERREW system (Erdik, Fahjan, et al., 2003). We will demonstrate the performance of PreSEIS using (1) a subset of simulated events, (2) the entire database, and (3) two scenario earthquakes.

\section{Results for a Subset of Simulations}

Let us first consider a subset of earthquakes in the synthetic database. Based on this subset we will demonstrate how we can improve the estimates of seismic source parameters by using different numbers of sensors and different lengths of the seismic signals. In other words, we will study the problem of incomplete information. Figure 3 a shows the epicenters of 31 simulated earthquakes in the Izmit Bay, which is in the eastern Marmara region. These events have been selected under the criterium that the $P$ wave reaches first either station FARGE or TUZ01. We train the TLFF networks with the seismic attributes of all $s=1, \ldots, 31$ earthquakes in the subset at seven timesteps between $t^{1}=0.5 \mathrm{sec}$ and $t^{7}=3.5 \mathrm{sec}$ after triggering of the first EEW sensor at $\tau_{i_{0}}$. Next, we evaluate the performance of PreSEIS with respect to the estimated source parameters $\left(\hat{\Phi}^{n}, \hat{\Theta}^{n}, \hat{Z}^{n}\right)$ and $\hat{M}^{n}$ using equations (3a) to (3c) and (5). In this first test we consider only the ideal data that are perfectly picked and that are not affected by noise.

The performance results shown in Figures $3 b$ and $3 c$ refer to the training data. Figure $3 \mathrm{~b}$ visualizes the absolute location errors

$$
\mathrm{AE}^{\mathrm{loc}, n}\left(\mathbf{w}^{\mathrm{loc}, n}\right) \equiv \sum_{s=1}^{S}\left|\left(\Phi_{s}, \Theta_{s}, Z_{s}\right)-\left(\hat{\Phi}_{s}^{n}, \hat{\Theta}_{s}^{n}, \hat{Z}_{s}^{n}\right)\right|
$$

for all $n=1, \ldots, 7$ timesteps. The performance of PreSEIS is quantified by the twenty-fifth, fiftieth, seventy-fifth, and ninety-fifth percentiles of the corresponding error distributions. For instance, the fiftieth percentile (also known as 
the median) divides the distribution in two halves, with 50\% of the earthquakes showing higher and 50\% showing smaller errors than given by the respective percentile. For the results shown in Figure 3b, from left to right, we derive the required seismic attributes $\Delta \tau_{i}^{n}$ and $\log \left(\mathrm{CAV}_{i}^{n}+1\right)$ from ground-motion observations at $I=2, I=3$, and $I=4$ sensors, respectively. Within the first $3.5 \mathrm{sec}$ after triggering $\tau_{i_{0}}$ the median location error can be reduced from 6 to $2 \mathrm{~km}$ using two sensors, from 3.5 to $1.5 \mathrm{~km}$ using three sensors, and from 3 to $1 \mathrm{~km}$ using four sensors. Recall from equation (1), that using two sensors, for instance, does not necessarily mean that the $P$ wave has already reached both sensors before estimates are issued. PreSEIS includes the information on nontriggered sensors to confine the space of possible solutions to the location problem.

Estimates of the moment magnitude $\mathbf{M}$ also exhibit a clear enhancement with time and with increasing number of sensors involved (Fig. 3c). The reliabilities of $\hat{M}^{n}$ at $0.5,1.5,2.5$, and $3.5 \mathrm{sec}$ (from left to right) after $\tau_{i_{0}}$, using $I=2, I=3$, and $I=4$ sensors (from top to bottom), are quantified (1) by the correlation coefficient $R$, which is defined by

$$
R^{n}\left(\mathbf{M}, \hat{M}^{n}\right) \equiv \frac{\operatorname{Cov}\left(\mathbf{M}, \hat{M}^{n}\right)}{\sqrt{\operatorname{Cov}(\mathbf{M}, \mathbf{M}) \operatorname{Cov}\left(\hat{M}^{n}, \hat{M}^{n}\right)}},
$$

with covariance matrices $\operatorname{Cov}(\cdot)$, and (2) by the standard deviation $\sigma$ of the errors given by

$$
\sigma^{n} \equiv \sqrt{\frac{1}{30} \sum_{s=1}^{31}\left(\left(\mathbf{M}_{s}-\hat{M}_{s}^{n}\right)-\frac{1}{31} \sum_{s^{\prime}=1}^{31}\left(\mathbf{M}_{s^{\prime}}-\hat{M}_{s^{\prime}}^{n}\right)\right)^{2}} .
$$

(a)

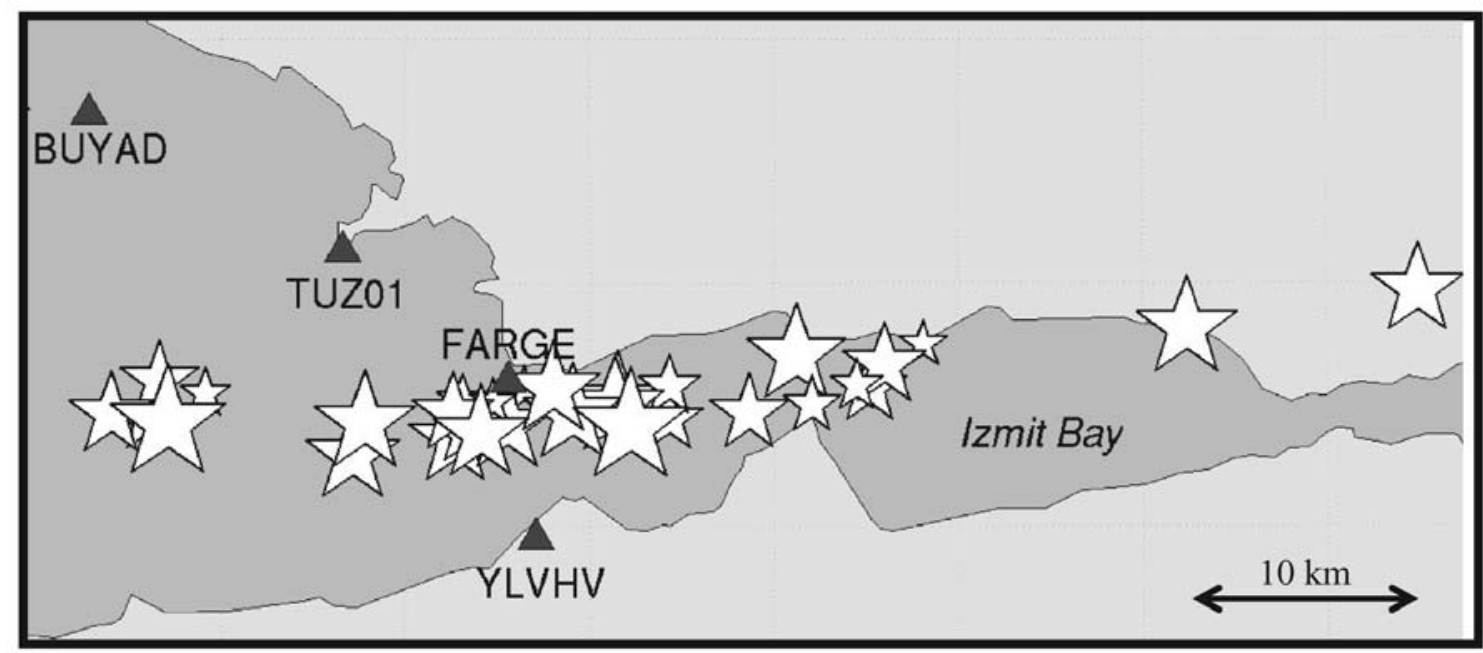

(b)
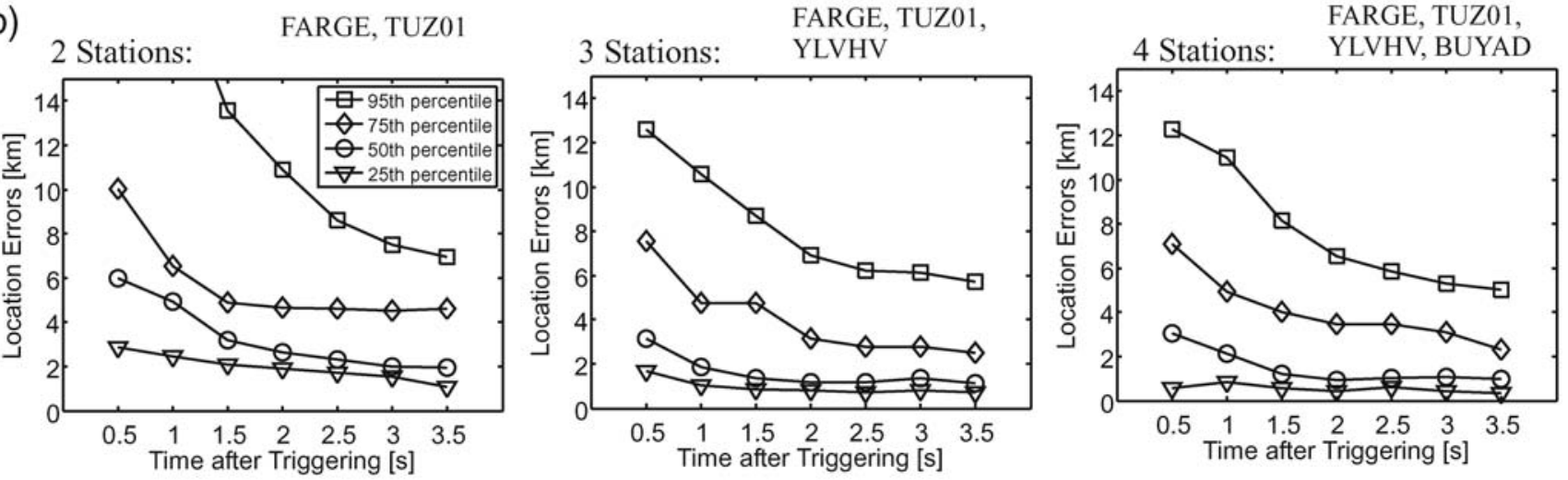

Figure 3. (a) Epicenters of 31 simulated earthquakes in the Izmit Bay in the eastern Marmara region. The events are a subset of the synthetic database in Figure 2. The triangles mark the locations of four EEW stations as part of the IERREWS. (b) Absolute errors of hypocenter locations $\mathrm{AE}^{\mathrm{loc}, n}\left(\mathbf{w}^{\mathrm{loc}, n}\right)$ for the events shown in (a), using, from left to right, the information on $P$-wave arrivals at two, three, and four sensors, respectively. The errors are quantified by the twenty-fifth, fiftieth, seventy-fifth, and ninety-fifth percentiles of the error distributions at timesteps between 0.5 and $3.5 \mathrm{sec}$ after $\tau_{i_{0}}$. (c) Observed versus estimated moment magnitudes, $\mathbf{M}$ and $\hat{M}$, at four timesteps (from left to right) using the CAV values at two, three, and four stations, respectively (from top to bottom). The accuracy of predicted magnitudes is quantified by the correlation coefficient $R$ and the standard deviation $\sigma$ of the errors. The results in (b) and (c) show a clear enhancement of estimates with time and the number of stations involved.

(Continued) 

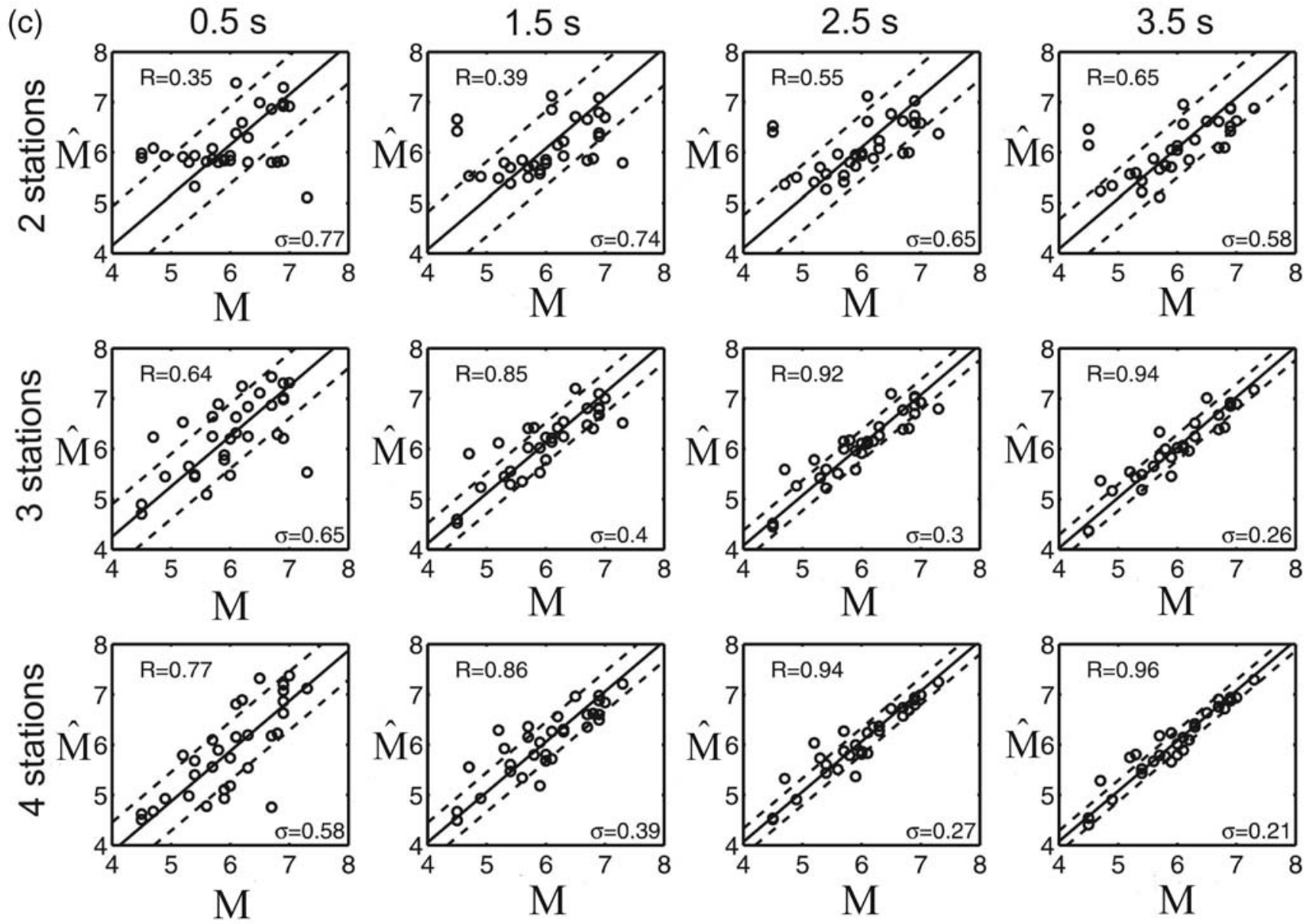

Figure 3. Continued.

While the prediction of $\mathbf{M}$ at $0.5 \mathrm{sec}$-based on the information at two sensors (Fig. 3c, top left)-is fairly poor $(R=0.35)$, it can be significantly improved, if one ( $R=0.64)$ or two further sensors $(R=0.77)$ are additionally involved. At $3.5 \mathrm{sec}$ after $\tau_{i_{0}}$, the prediction accuracy increases significantly if two $(R=0.65)$, three ( $R=0.94)$, and four sensors $(R=0.96)$ are used.

These tests demonstrate that estimates of seismic source parameters clearly are enhanced with elapsed time and the number of stations involved. At the same time, this observation reveals a general trade-off in EEW: at each timestep after the detection of an earthquake we will only be capable to specify its source parameters (and thus levels of warnings) to a certain degree of reliability. The longer we wait, the better the predictions, but the shorter the remaining warning times.

\section{Results for the Entire Database}

We now split the database of all 280 scenarios at random into one training $(70 \%)$, one test $(20 \%)$, and one validation subset $(10 \%)$. The 10 perturbations of each scenario are assigned to the same subset as the scenario itself. The valida- tion and test subsets are used for the evaluation of network performance during and after the training. With help of the validation subset we determine the iteration step at which we terminate the training of the ANNs as described before. In order to test the stability of the obtained results, we repeat the training and evaluation procedures several times. Each time we randomly interchange training, test, and validation subsets (Efron and Tibshirani, 1993), and also use different weight initializations of the TLFF networks at the beginning of the training procedure.

Figure 4 summarizes the performance of PreSEIS for the training and test patterns in the database as a function of time after $\tau_{i_{0}}$. Again, we quantify the absolute errors of the hypocenter locations $\mathrm{AE}^{\mathrm{loc}, n}\left(\mathbf{w}^{\mathrm{loc}, n}\right)$, defined in equation (15), by the twenty-fifth, fiftieth, seventy-fifth, and ninety-fifth percentiles (Fig. 4, left column). The magnitude errors are characterized by the mean and the standard deviation of prediction errors

$$
\mathrm{E}^{\mathrm{mag}, n}\left(\mathbf{w}^{\mathrm{mag}, n}\right)=\sum_{s=1}^{S}\left(\mathbf{M}_{s}-\hat{M}_{s}^{n}\right)
$$

(Fig. 4, middle column). We test the sensitivity of PreSEIS to nonperfect input information by usage of the noisy data that 

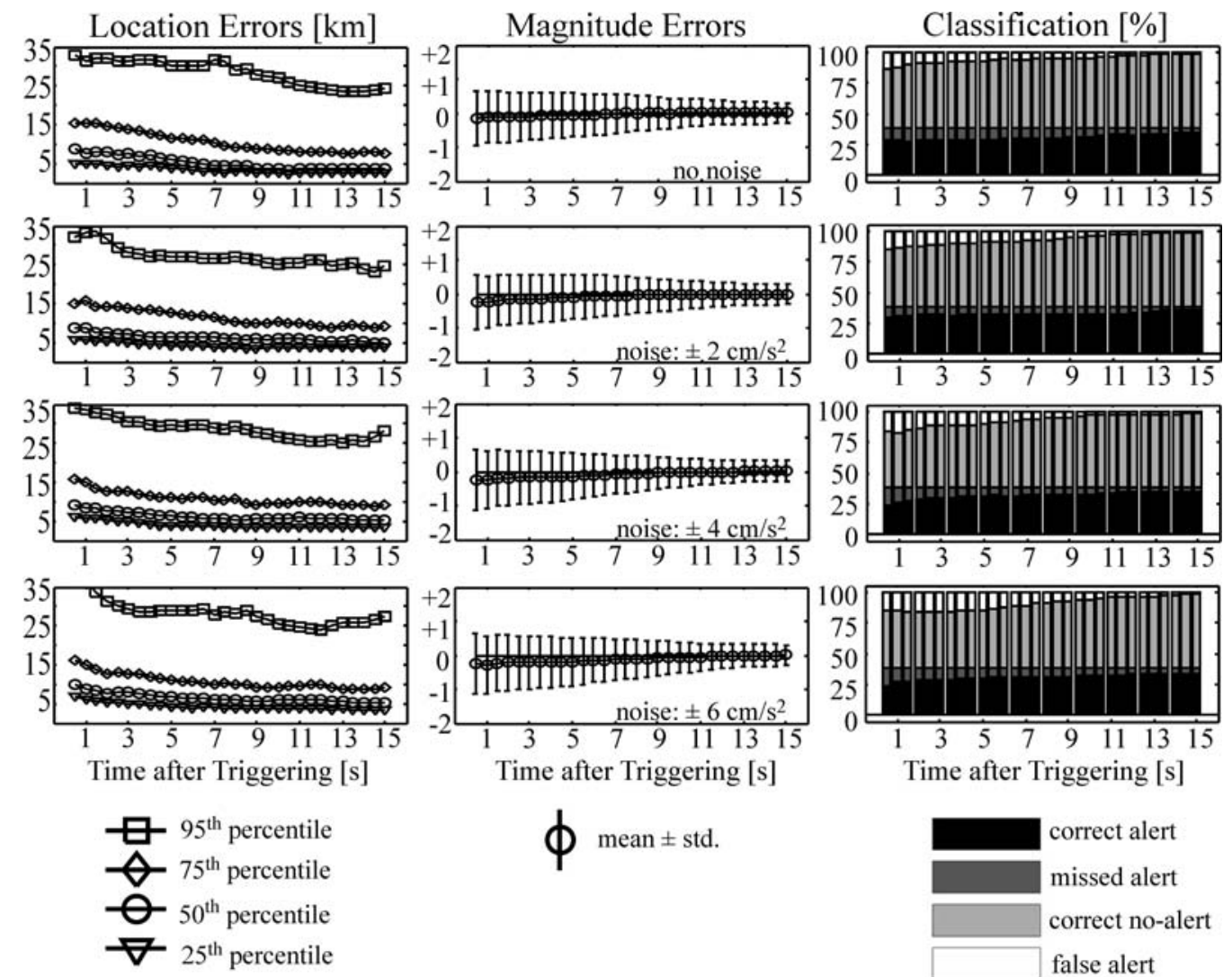

$\oint$ mean \pm std.

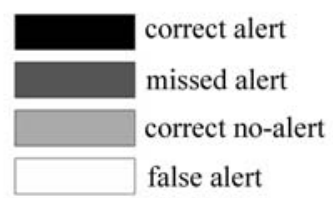

Figure 4. Results for all 280 simulated earthquake scenarios and their 2800 perturbations. From left to right: location errors, magnitude errors, and classification performance, each as a function of time after $\tau_{i_{0}}$. The hypocenter location errors $\mathrm{AE}^{\mathrm{loc}, n}\left(\mathbf{w}^{\mathrm{loc}, n}\right)$ (left column) are quantified by the twenty-fifth, fiftieth, seventy-fifth, and ninety-fifth percentiles of the error distributions. Fifty percent of the scenarios have location errors of less than $10 \mathrm{~km} 0.5 \mathrm{sec}$ after $\tau_{i_{0}}$ and less than $5 \mathrm{~km}$ after $15.0 \mathrm{sec}$. The mean magnitude error $\mathrm{E}^{\mathrm{mag}, n}\left(\mathbf{w}^{\mathrm{mag}, n}\right)(\mathrm{middle}$ column) is zero at all timesteps for the ideal data (top row), and -0.2 units for the data if affected by Gaussian noise with amplitudes of \pm 2 , \pm 4 , and $\pm 6 \mathrm{~cm} / \mathrm{sec}^{2}$ (rows 2 to 4 ). The standard deviation of the errors is 0.8 units after $0.5 \mathrm{sec}, 0.5$ units after $7.5 \mathrm{sec}$, and 0.3 after $15.0 \mathrm{sec}$. Inserting $\left(\hat{\Phi}^{n}, \hat{\Theta}^{n}, \hat{Z}^{n}\right)$ and $\hat{M}^{n}$ into the empirical attenuation law (equation 14b) allows predicting the level of ground shaking at site UserX. Every fourth damaging earthquake with $M M I \geq \mathrm{VI}$ at this site is missed after $1.0 \mathrm{sec}$, every fifth after 7.5 sec, and every fifteenth after $15.0 \mathrm{sec}$. Every fourth to fifth warning is incorrect $1.0 \mathrm{sec}$ after $\tau_{i_{0}}$, every tenth after $7.5 \mathrm{sec}$, and every hundredth after $15.0 \mathrm{sec}$. In the case of noisy input data (rows 2 to 4 ) the rate of misclassifications increases by $1 \%$ to $2 \%$.

was described in the previous section. Figure 4 shows the performance results for noise amplitudes of $\pm 0, \pm 2, \pm 4$, and $\pm 6 \mathrm{~cm} / \mathrm{sec}^{2}$ (from top to bottom), respectively.

As in the previous study we see a clear increase in the reliability of $\left(\hat{\Phi}^{n}, \hat{\Theta}^{n}, \hat{Z}^{n}\right)$ and $\hat{M}^{n}$ with time. The median of the location errors decreases from less than $10 \mathrm{~km}$ at $0.5 \mathrm{sec}$ after $\tau_{i_{0}}$ to less than $5 \mathrm{~km}$ during the subsequent $15.0-\mathrm{sec}$ interval; the ninety-fifth percentile generally starts with 30 to $35 \mathrm{~km}$ and falls to about $25 \mathrm{~km}$. These errors are mainly linked to earthquakes that occur beyond the fault segments or at the border of the sensor network. The highest observed location error is $90 \mathrm{~km}$. The mean error of $\hat{M}^{n}$ for the ideal data is zero at all timesteps $n$ (Fig. 4, middle column, top row). The different noise levels (rows 2 to 4 ) lead to an overestimation of the magnitudes by 0.2 units on average in the first few seconds. For both the ideal and the noisy data, we observe a decrease of $\sigma$ from 0.8 to 0.3 magnitude units in the analyzed 15.0-sec interval. All results are stable and hardly show any dependence on either the Bootstrap procedure or the weight initialization.

As we have simulated the seismic ground motions not only at the EEW sites, but also at site UserX as a possible recipient of warnings, we can use the empirical FAS-MMI relation after Sokolov (2002) referred to earlier and classify all 280 earthquake scenarios with respect to their (true) $M M I$ level at this site. For EEW we estimate $\hat{M}^{n}$ at timestep $n$ from equation (14b) and round this value to the next full intensity level. We set the following task: whenever the rounded intensity is expected to exceed VI units at site UserX, PreSEIS shall issue a warning. We choose this fairly low threshold to assure a well-balanced distribution of alarm (40\%) and nonalarm $(60 \%)$ events. Using the previously established definitions of (1) correct alerts, (2) missed alerts, (3) correct no alerts, and (4) false alerts, we observe, once again, a clear improvement of predictions with time. For the ideal data (top row), that is, without noise, the rate of false alerts is 
$14 \%$ within the first seconds after $\tau_{i_{0}}, 6 \%$ after $7.5 \mathrm{sec}$, and less than $1 \%$ after $15.0 \mathrm{sec}$. This means that $1.0 \mathrm{sec}$ after $\tau_{i_{0}}$ every fourth to fifth warning is incorrect. The rate decreases to every tenth after $7.5 \mathrm{sec}$ and to every hundredth after $15.0 \mathrm{sec}$. The rate of missed alerts is $10 \%, 8 \%$, and $3 \%$, respectively. This means that every fourth damaging earthquake is missed after $1.0 \mathrm{sec}$, every fifth after $7.5 \mathrm{sec}$, and every fifteenth after $15.0 \mathrm{sec}$. In the case of noisy input data (Fig. 4, right column, rows 2 to 4 ) the rates of misclassifications increase by merely $1 \%$ to $2 \%$.

\section{Results for Two Scenario Earthquakes}

In the following, we will analyze the results for two earthquake scenarios with moment magnitudes $\mathbf{M} 7.3$ in more detail. Both earthquakes are accompanied by $80 \mathrm{~km}$ long ruptures of the western fault in the Sea of Marmara (Fig. 5) with epicentral distances of approximately $75 \mathrm{~km}$ to Istanbul. The modelling parameters of the two scenarios are exactly the same, only their slip distributions differ: for scenario 1 we assume that the largest fault slip of approximately $10 \mathrm{~m}$ occurs close to the earthquake hypocenter (Fig. 5a, left). For scenario 2 we assume that the largest slip occurs about $20 \mathrm{~km}$ away (Fig. 5a, right). Because the moment magnitude, by definition, depends on the average slip on the rupture area, very distinct slip distributions will lead to the same magnitude level. The simulated ground motions at the 10 EEW sensors and site UserX are shown in Figure 5b. For the velocity model with $\alpha=5.7 \mathrm{~km} / \mathrm{sec}$ used here, the $P$ wave will arrive at station BOTAS approximately $4.0 \mathrm{sec}$ after the rupture initiation. The maximum warning times for the two scenarios, which are defined by the time window between the $P$-wave detection at BOTAS and the $S$-wave arrivals in the Marmara region, are less than $25 \mathrm{sec}$ (Fig. 5c).

Figure $5 \mathrm{~d}$ illustrates the predictions of the moment magnitudes for both scenarios, starting $0.5 \mathrm{sec}$ after the triggering of station BOTAS. Every half second the estimates are up- dated using the available seismic attributes at all 10 sensors. Note that already $1.0 \mathrm{sec}$ after triggering $\tau_{i_{0}}$, PreSEIS recognizes the earthquake to be strong with $\hat{M}>6.5$. That is, the event is likely to be damaging and a warning is necessary. With ongoing time, $\hat{M}$ increases and shows a clear convergence towards the true magnitude $\mathbf{M}$ 7.3. Note that for almost all timesteps the estimates for scenario 1 are at a higher level than those for scenario 2. This behavior results from the largest slip occurring close to the point of rupture initiation in scenario 1 , which means that the largest energy is radiated within the first few seconds (Fig. 5a, left). Consider, for example, the first six seconds of the seismic signals at station BOTAS. These parts of the signals carry information on the seismic rupture that are marked in Figure 5a by the segments of two circles: in scenario 1 the largest slip has already occurred, while in scenario 2 the largest slip has not yet happened. Consequently, we observe a good fit between $\hat{M}$ and $\mathbf{M} 6.0 \mathrm{sec}$ after $\tau_{i_{0}}$ for scenario 1 and an underestimation for scenario 2 (Fig. 5d). Our example demonstrates the importance of steady updates of estimates of seismic source parameters in algorithms for EEW: these updates are essential for the applicability to complex rupture histories.

The level and distribution of $M M I$ intensities are estimated for both scenarios from simulated ground motions applying the stochastic simulation method for finite faults (SSMFF) (Beresnev and Atkinson, 1997) at a large number of sites on a regular grid of $0.1^{\circ}$ spacing. Using, once again, the empirical relationship between the FAS and MMI intensities (Sokolov, 2002), we calculate the corresponding intensities and interpolate these values to obtain shake maps (Fig. 6a). Note the different expansions of the strong-motion centroids due to the distinct slip distributions of the two scenarios (Fig. 5a). The intensities range between IX and $X$ close to the seismic rupture and between IV and VIII onshore. Note from Figure 6a that the SSMFF is incapable of reproducing directivity effects.
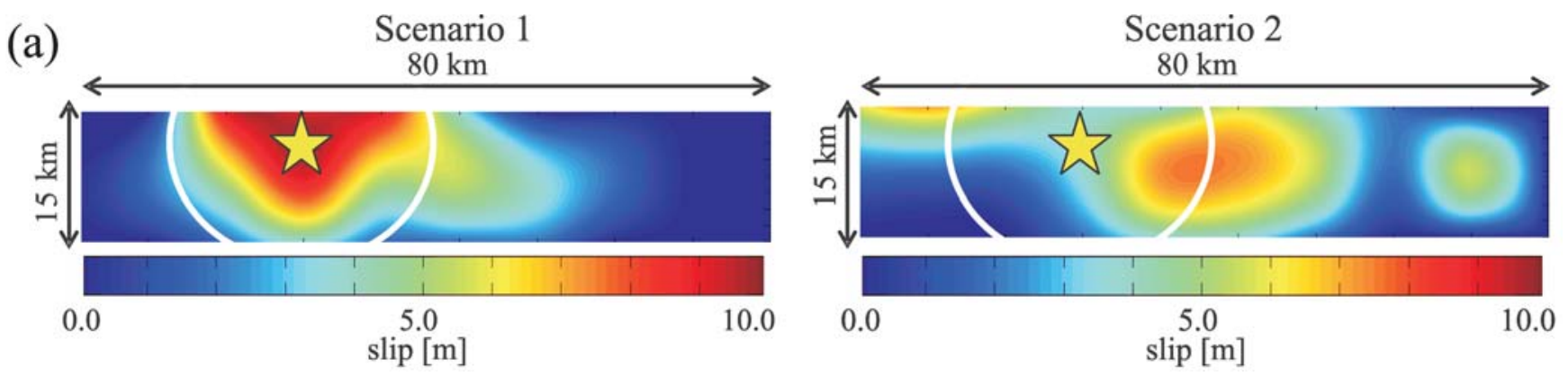

Figure 5. (a) Slip distributions for two scenario earthquakes along the western fault with $\mathbf{M}$ 7.3. The largest slip in scenario 1 (left) occurs close to the point of rupture initiation, in scenario 2 (right), approximately $20 \mathrm{~km}$ away. The segments of the two circles mark the boundary between ruptured and nonruptured parts of the faults about $5 \mathrm{sec}$ after the rupture initiation. (b) Mean horizontal components of simulated ground-motion time series at the 10 EEW stations of the IERREWS. The bars indicate $P$ - and $S$-wave onsets. (c) Warning times in the Marmara region for the two scenarios, which are defined by the time difference between the $P$-wave detection at station BOTAS and $S$-wave arrivals at the different sites. For the Istanbul metropolitan area we expect warning times between 12 and 22 sec. (d) Predicted moment magnitudes $\hat{M}$ for both scenarios as a function of time after the triggering of BOTAS. The convergence towards the true magnitude M 7.3 (dashed line) is faster for scenario 1 than for scenario 2 because most of the seismic energy is radiated within the first few seconds.

(Continued) 
(b)
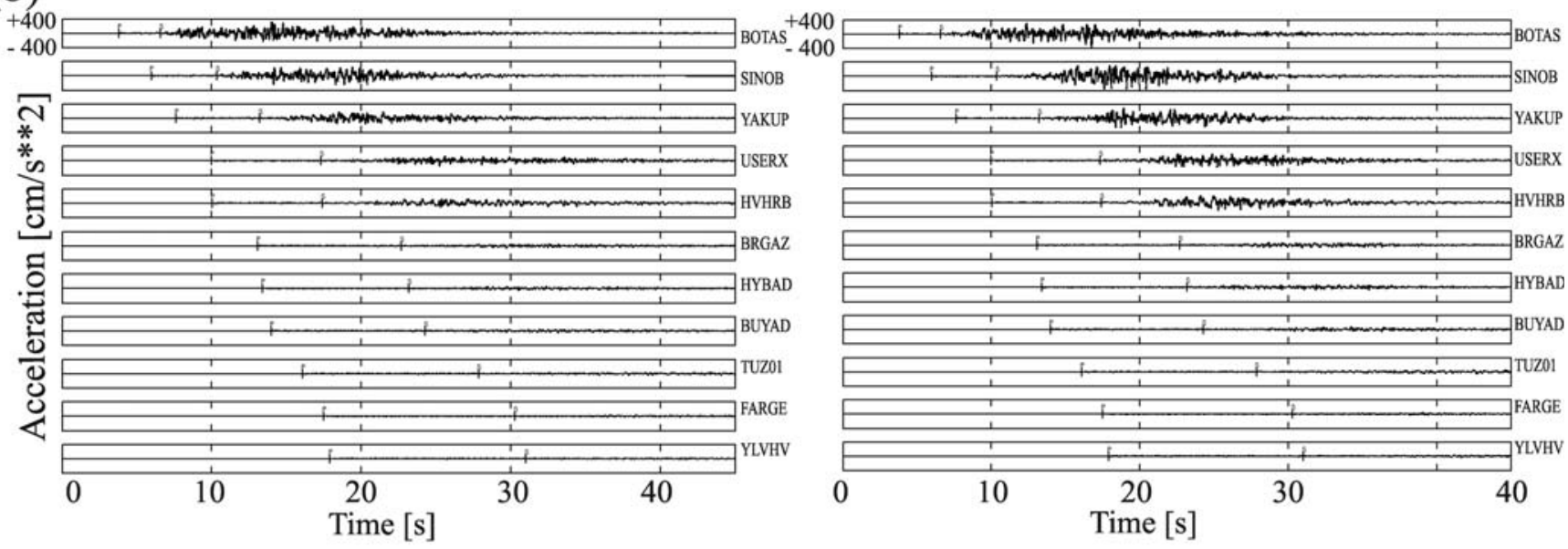

(c)

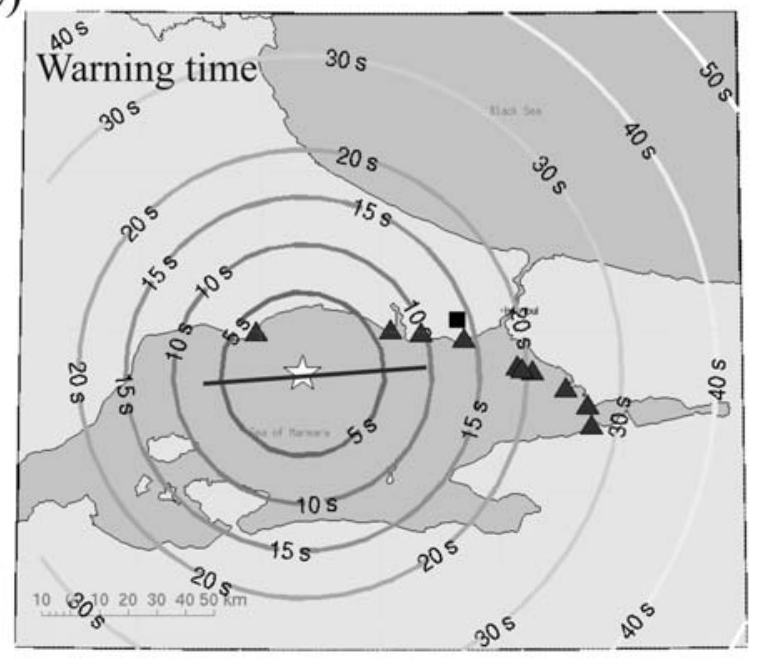

(d)

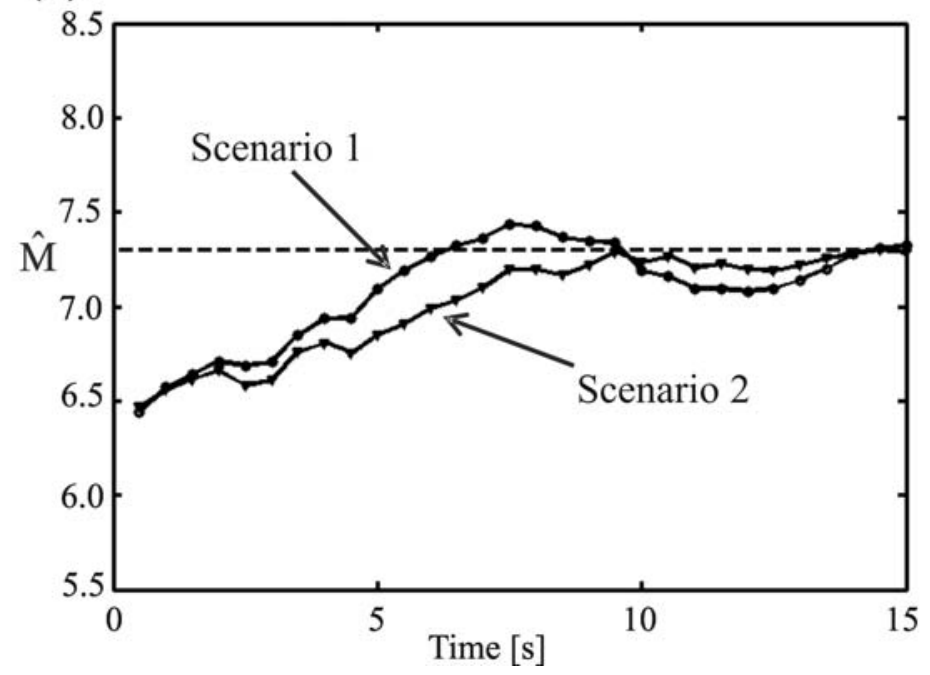

Figure 5. Continued.

As described before, we can approximate the shake maps by alert maps by inserting the estimated source parameters $\hat{M}^{n}$ and $\left(\hat{\Phi}_{\text {start }}^{n}, \hat{\Theta}_{\text {start }}^{n}, \hat{\Phi}_{\text {end }}^{n}, \hat{\Theta}_{\text {end }}^{n}\right)$ into equation (14b). Figure $6 \mathrm{~b}$ visualizes four alert maps for $M M I$ in the Marmara region for the two scenario earthquakes at timesteps between 0.5 and $7.5 \mathrm{sec}$ after $\tau_{i_{0}}$ (from top to bottom). Note that, as the shake and alert maps in Figures $6 \mathrm{a}$ and $6 \mathrm{~b}$ have been calculated for rock condition, they might underestimate $M M I$ in the case of strong site effects due to local soil conditions as well as to directivity effects.

In the ideal case, shake and alert maps do not differ much from each other. The comparison of the different maps in Figures $6 \mathrm{a}$ and $6 \mathrm{~b}$ reveals that initially the ground shaking in the Marmara region is underestimated by approximately one intensity unit, although for both scenarios the predictions exhibit a clear convergence towards the true levels between IV and VIII. Alert maps, as presented here, could have been made public between 7 and $17 \mathrm{sec}$ before strong shaking in the Istanbul metropolitan area will occur (see Fig. 5c).

\section{Discussion and Conclusions}

We have developed a new method for EEW that is based on two-layer feed-forward (TLFF) neural networks and that is applicable to finite fault. PreSEIS inverts time-dependent seismic attributes that are derived from ground-motion observations at different sensors in a seismic network. At regular timesteps after the triggering of the first EEW sensor by the propagating seismic $P$ wave, PreSEIS estimates the most likely hypocenter location, moment magnitude, and rupture expansion of an earthquake in progress. Our studies demonstrate a clear and fast convergence of these estimates towards correct solutions with time. PreSEIS achieves a robust performance and, at the same time, issues first estimates only $0.5 \mathrm{sec}$ after the triggering of the first sensors (i.e., is as fast as onsite warning approaches). 
The capability to issue estimates (and thus warnings) as soon as the $P$ wave reaches the first sensor in the network results from two sources. First, PreSEIS includes the information that the $P$ wave has not reached certain sensors, which means that we can rule out certain positions of the hypocenter (Rydelek and Pujol, 2004; Horiuchi et al., 2005). Second, PreSEIS uses the information on likely source locations: the ANNs have learned from experience (i.e., from the training examples) that earthquakes generally occur along major faults. Hence, there are certain source locations that are more likely than others.

The optimum response to a warning will depend on user-specific needs and costs in the case of destructive earthquakes or false alerts, respectively. The knowledge of uncertainties of predictions at the different timesteps, as specified in this article, will provide important decision-making information for optimum response of each user. Our studies reveal a trade-off between the prediction accuracy on the one hand, and the remaining warning time on the other hand.

ANNs have the advantage that they can handle nonlinear and noisy data. However, they require a comprehensive database of training examples, from which they can learn the associations between seismic observations and source parameters. Note that ANNs are good in interpolations of (nonlinear) data, but they reveal uncertainties if applied for extrapolation (Swingler, 1996). This means that the behavior of an ANN is hardly predictable for earthquake scenarios with source characteristics very distinct from those of previous events.

PreSEIS requires no assumptions on rupture determinism unlike other methods for EEW, such as those based on the predominant period of the initial part of the seismic records as an indicator for the earthquake magnitude (Nakamura, 1989; Allen and Kanamori, 2003; Olson and Allen, 2005). As we have demonstrated in this article, PreSEIS does not assume that the largest slips on the faults necessarily occur close to the point of rupture initiation. Because of its integration of the entire rupture history of an earthquake (by the calculation of the CAV using equation 2), the method can also handle complex ruptures.

PreSEIS is a method for EEW for finite faults. In addition to the estimates $\hat{M}^{n}$ and $\left(\hat{\Phi}^{n}, \hat{\Theta}^{n}, \hat{Z}^{n}\right)$ at timestep $n$, it allows to predict the expansion of the evolving earthquake rupture, or more strictly, the expansion of the strong-motion centroid described by $\left(\hat{\Phi}_{\text {start }}^{n}, \hat{\Theta}_{\text {start }}^{n}, \hat{\Phi}_{\text {end }}^{n}, \hat{\Theta}_{\text {end }}^{n}\right)$. This information is useful for replacing common point source measures, such as the hypocentral distance, in the empirical attenuation laws (see equation 10a) by Joyner-Boore distances. This will clearly help to reflect the shapes of intensity distributions in alert maps in a much more realistic way. The information on rupture expansions is also critical for the estimation of directivity effects. Moreover, these effects can be integrated into attenuation laws, as shown by Somerville et al. (1997), and therewith, once again, into the alert maps. However, we could not test this idea here, because our simulated acceleration time series do not exhibit directivity effects (Fig. 6a).

PreSEIS assumes a properly working network of seismic sensors. If applied to real data streams, we will not encounter this perfect situation. The recorded data at the sensors will be noisy and some of the sensors may fail. Recall, however, that PreSEIS is based on an ANN formulation, which has a very high tolerance of noisy data. We have tested this behavior through the perturbation of the ideal data by

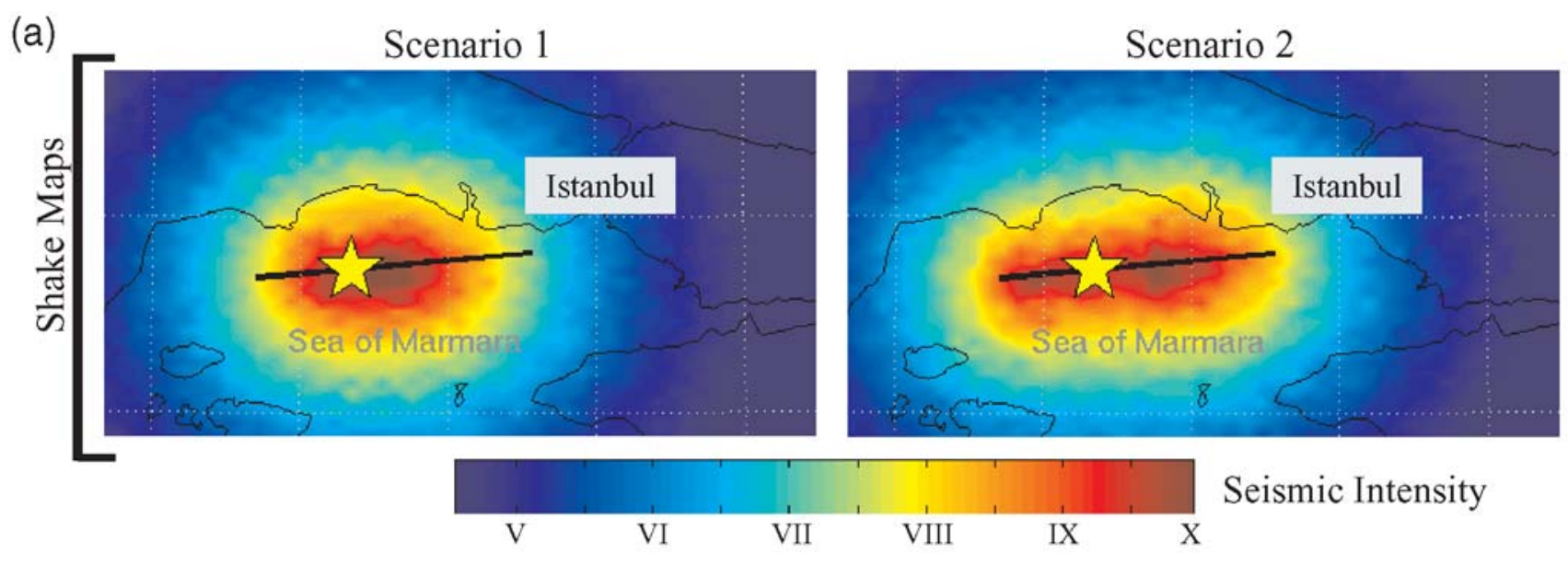

Figure 6. Shake (a) and alert maps (b) for the two scenario earthquakes in Figure 5. (a) The distribution of seismic intensity is estimated from interpolated $M M I$ values. These are obtained from the FAS of seismic simulations on a regular grid of $0.1^{\circ}$ spacing using the empirical FAS-MMI relation proposed by Sokolov (2002). (b) The curves of equal intensity (isoseists) in the alert maps for scenario 1 (left) and scenario 2 (right) are determined from the empirical attenuation law for rock condition in equation (14b) at four timesteps between 0.5 and $7.5 \mathrm{sec}$ after $\tau_{i_{0}}$. The fronts of $P$ and $S$ waves are indicated by solid and dashed lines; the true and estimated epicenter locations are marked by stars and circles. PreSEIS initially underestimates the $M M I$ levels of both scenarios by approximately one intensity unit, but exhibits a fast convergence towards the target shake maps in (a) as time increases.

(Continued) 

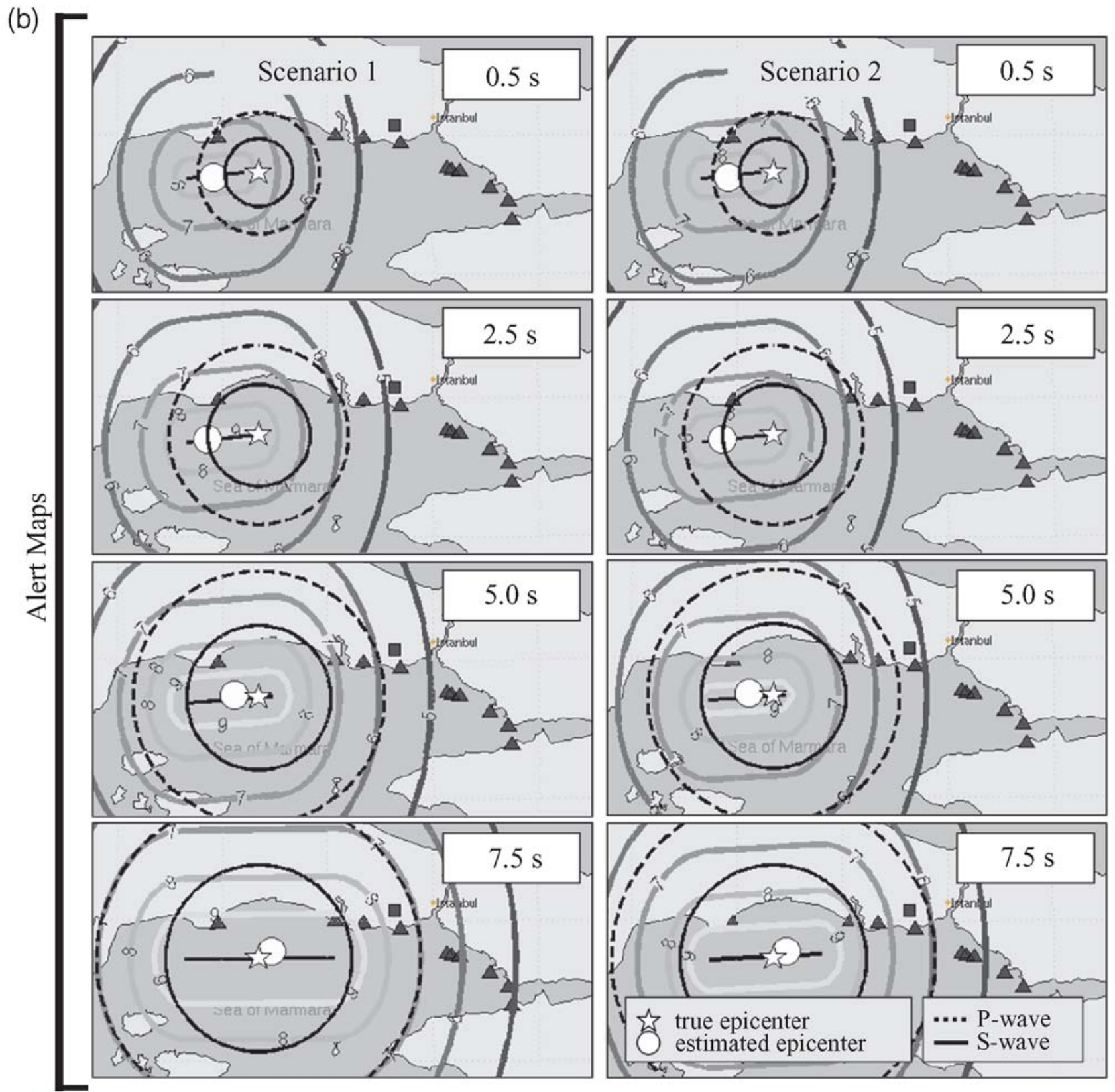

Figure 6. Continued.

adding different levels of Gaussian noise. The mean magnitude error increases by merely 0.2 units. The problem of failing instruments during operation can be solved, for example, by different combinations and subsets of sensors for which different ANNs have been trained. During operation, the EEW system then would simply require the information, which of the sensors is disrupted. It would then choose the respective ANN that does not require the information at this sensor to estimate the source parameters of the current earthquake.

The main objective of this article is the (theoretical) development and demonstration of a new approach to EEW rather than the design of a final tool that will be applicable to real data. Our studies are based on a database of simulated ground-motion time series for finite faults. We have tried to make these simulations as realistic as possible by usage of regional information on the underground in the Marmara region and random variability of source parameters. In addition, Böse (2006) determined different ground-motion parameters (peak values, seismic intensity, and spectral response) from the simulated data and compared these, (1) with observations during the 1999 Kocaeli M 7.4, the 1999 M 7.2 Düzce, and the $2004 M_{\mathrm{L}} 4.3$ Yalova earthquakes in Turkey, and (2) with empirical attenuation laws published by Erdik et al. (1985), Boore et al. (1997), Campbell (1997), and Sa- 
digh et al. (1997). These comparisons exhibit a good agreement between the simulated and the observational data. We conclude that our stochastic simulations reflect the seismic ground motions in the Marmara region in a reasonable way. However, we are aware of possible limitations resulting from the use of synthetic data, for example, with respect to the likely underestimation of site effects. In the future we will therefore test and extend the PreSEIS approach by using combined datasets of observational and simulated data from California and Japan.

\section{Acknowledgments}

The authors are very grateful for the helpful comments of Jose Pujol and two anonymous reviewers. This study was supported by the Collaborative Research Center Grant Number 461: "Strong Earthquakes-A Challenge for Geosciences and Civil Engineering" at the Universität Karlsruhe (TH), Germany, funded by the Deutsche Forschungsgemeinschaft (German Research Foundation) and the State of Baden-Württemberg, Germany.

\section{References}

Allen, R. (2007). The ElarmS earthquake early warning methodology and application across California, in Earthquake Early Warning Systems, P. Gaspirini, G. Manfredi and J. Zschau (Editors) Springer, New York, 21-43.

Allen, R., and H. Kanamori (2003). The potential for earthquake early warning in southern California, Science 300, 786-789.

Armijo, R., B. Meyer, S. Navarro, G. King, and A. Barka (2002). Asymmetric slip partitioning in the Sea of Marmara pull-apart: a clue to propagation processes of the North Anatolian fault?, Terra Nova 14, no. 2, $80-86$.

Atkinson, G., and D. Boore (1995). Ground motion relations for eastern North America, Bull. Seismol. Soc. Am. 85, no. 1, 17-30.

Beresnev, I. (2001). What we can learn and cannot learn about earthquake sources from the spectra of seismic waves, Bull. Seismol. Soc. Am. 91, no. 2, 397-400.

Beresnev, I., and G. Atkinson (1997). Modeling finite-fault radiation from the $\omega^{\mathrm{n}}$ spectrum, Bull. Seismol. Soc. Am. 87, no. 1, 67-84.

Bishop, C. (1995). Neural Networks for Pattern Recognition, Clarendon, London.

Boore, D. (1983). Stochastic simulation of high-frequency ground motions based on seismological models of the radiated spectra, Bull. Seismol. Soc. Am. 73, 1865-1894.

Boore, D. (1986). Short-period P- and S-wave radiation from large earthquakes, Bull. Seismol. Soc. Am. 76, no. 1, 1865-1894.

Boore, D. (2003). Simulation of ground motion using the stochastic method, Pure Appl. Geophys. 160, 635-676.

Boore, D., and J. Boatwright (1984). Average body-wave radiation coefficients, Bull. Seismol. Soc. Am. 74, 1615-1621.

Boore, D., and W. Joyner (1997). Site amplifications for generic rock studies, Bull. Seismol. Soc. Am. 87, no. 2, 327-341.

Boore, D., W. Joyner, and T. Fumal (1997). Equations for estimating horizontal response spectra and peak acceleration from western North American earthquakes: a summary of recent work, Seism. Res. Lett. 68, no. 1, 128-153.

Böse, M. (2006). Earthquake early warning for Istanbul using artificial neural networks, Ph.D. Thesis, Karlsruhe University, $181 \mathrm{pp}$.

Böse, M., C. Ionescu, and F. Wenzel (2007). Earthquake early warning for Bucharest, Romania: novel and revised scaling relations, Geophys. Res. Lett. 34, L07302, doi 10.1029/2007GL029396.

Brune, J. (1970). Tectonic stress and the spectra of seismic shear waves from earthquakes, J. Geophys.Res. 75, 4997-5009.

Brune, J. (1971). Correction, J. Geophys. Res. 76, 5002.
Campbell, K. (1997). Empirical near-source attenuation relations for horizontal and vertical components of peak ground acceleration, peak ground velocity, and pseudo-absolute acceleration response spectra, Seism. Res. Lett. 68, no. 1, 154-179.

Efron, B., and R. J. Tibshirani (1993). An Introduction to the Bootstrap, Chapman and Hall, London, 436 pp.

Erdik, M., N. Aydinoglu, Y. Fahjan, K. Sesetyan, M. Demircioglu, B. Siyahi, E. Durukal, C. Ozbey, Y. Biro, H. Akman, and O. Yuzugullu (2003). Earthquake risk assessment for Istanbul metropolitan area, Earthq. Eng. Eng. Vib. 2, no. 1, 1-23.

Erdik, M., V. Doyoran, N. Akkas, and P. Gulkan (1985). A probabilistic assessment of the seismic hazard in Turkey, Tectonophysics 117, 295-344.

Erdik, M., Y. Fahjan, O. Ozel, H. Alcik, A. Mert, and M. Gul (2003). Istanbul earthquake rapid response and the early warning system, Bull. Earthq. Eng. 1, 157-163.

Goltz, J. (2002). Report to OES and the operational areas: Introducing earthquake early warning in California: a summary of social science and public policy issues, Caltech Seismological Laboratory, Disaster Assistance Division.

Harben, P. (1991). Technical Report UCRL-LR-109625: Earthquake alert system feasibility study, Lawrence Livermore National Laboratory, Livermore, California.

Horasan, G., A. Kaslilar-Özcan, A. Boztepe-Güney, and N. Türkelli (1998). S-wave attenuation in the Marmara region, northwestern Turkey, Geophys. Res. Lett. 25, no. 14, 2733-2736.

Horiuchi, S., H. Negishi, K. Abe, A. Kamimura, and Y. Fujinawa (2005). An automatic processing system for broadcasting earthquake alarms, Bull. Seismol. Soc. Am. 95, no. 2, 708-781.

Jiang, J., G. Baird, and D. Blair (1998). Polarization and amplitude attributes of reflected plane and spherical waves, Geophys. J. Int. 132, $577-583$.

Kanamori, H. (2005). Real-time seismology and earthquake damage mitigation, Ann. Rev. Earth Planet. Sci. 33, 5.1.-5.20.

Kramer, S. (1996). Geotechnical Earthquake Engineering, International Series in Civil Engineering and Engineering Mechanics, Prentice Hall, New York.

Le Pichon, X., N. Chamot-Rooke, C. Rangin, and A. M. C. Sengor (2003). The North Anatolian fault in the Sea of Marmara, J. Geophys. Res. 108, no. B4, 2179, doi 10.1029/2002JB001862.

Levenberg, K. (1944). A method for the solution of certain non-linear problems in least squares, Q. J. Appl. Math., 2, 164-168.

Lockman, A., and R. Allen (2005). Single-station earthquake characterization for early warning, Bull. Seismol. Soc. Am. 95, no. 6, 2029-2039, doi $10.1785 / 0120040241$.

Nakamura, Y. (1989). Earthquake alarm system for Japan railways, Japan. Railw. Eng. 28, no. 4, 3-7.

Olson, E., and R. M. Allen (2005). The deteministic nature of earthquake rupture, Nature 438, 212-215, doi 10.1038/nature04214.

Parsons, T. (2004). Recalculated probability of $M \geq 7$ earthquakes beneath the Sea of Marmara, Turkey, J. Geophys. Res. 109, B05304, doi 10.1029/2003JB002667.

Pulido, N., A. Ojeda, K. Atakan, and T. Kubo (2004). Strong ground motion estimation in the Marmara Sea region (Turkey) based on a scenario earthquake, Tectonophysics 391, 357-374.

Rumelhart, D., G. Hinton, and R. Williams (1986). Parallel Distributed Processing: Explorations in the Microstructure of Cognition, Vol. 1, MIT Press, Cambridge.

Rydelek, P., and S. Horiuchi (2006). Is the earthquake rupture deterministic?, Nature 442, E5-E6, doi 10.1038/nature04963.

Rydelek, P., and J. Pujol (2004). Real-time seismic warning with a 2-station subarray, Bull. Seismol. Soc. Am. 94, 1546-1550.

Sadigh, K., C.-Y. Chang, J. Egan, F. Makdisi, and R. Youngs (1997). Attenuation relationships for shallow crustal earthquakes based on $\mathrm{Ca}-$ lifornia strong motion data, Seism. Res. Lett. 68, no. 1, 180-189.

Sokolov, V. (2002). Seismic intensity and Fourier acceleration spectra: revised relationship, Earthq. Spectra 18, no. 1, 161-187. 
Somerville, P. G., N. F. Smith, R. W. Graves, and N. A. Abrahamson (1997). Modification of empirical strong ground motion attenuation relations to include the amplitude and duration effects of rupture directivity, $\mathrm{Se}$ ism. Res. Lett. 68, 199-222.

Swingler, K. (1996). Applying Neural Networks: A Practical Guide, Academic, New York.

Wald, D., V. Quitoriano, T. Heaton, H. Kanamori, C. Scrivner, and C. Worden (1999). TriNet ShakeMaps: rapid generation of instrumental ground motion and intensity maps for earthquakes in southern California, Earthq. Spectra 15, 537-556.

Wenzel, F., M. Oncescu, M. Baur, and F. Fiedrich (1999). An early warning system for Bucharest, Seism. Res. Lett. 70, no. 2, 161-169.

Wu, Y.-M., and H. Kanamori (2005). Rapid assessment of damaging potential of earthquakes in Taiwan from the beginning of $\mathrm{P}$ waves, Bull. Seismol. Soc. Am. 95, 1181-1185.

Wu, Y.-M., and T.-1. Teng (2002). A virtual subnetwork approach to earthquake early warning, Bull. Seismol. Soc. Am. 92, no. 5, 2008-2018.
Geophysical Institute

Karlsruhe University

Hertzstrasse 16

76187 Karlsruhe, Germany

friedemann.wenzel@gpi.uni-karlsruhe.de

(M.B., F.W.)

Kandilli Observatory and Earthquake Research Institute

Bogazici University

Department of Earthquake Engineering

34684 Cengelkoy, Istanbul, Turkey

erdik@boun.edu.tr

(M.E.)

Manuscript received 3 January 2007 